\title{
LOCALLY HOMOGENEOUS AFFINE HYPERSPHERES WITH CONSTANT SECTIONAL CURVATURE
}

\author{
CECE LI
}

\begin{abstract}
In this paper, we study the $n$-dimensional locally homogeneous affine hyperspheres with constant sectional curvature, vanishing Pick invariant and the difference tensor $K$ satisfying $K^{n-1} \neq 0$. As main results, we classify such hyperspheres for dimension $n \leq 5$.
\end{abstract}

\section{Introduction}

An important problem in unimodular-affine differential geometry is to classify all the affine hyperspheres with affine metric of constant sectional curvature. The following results are well known.

THEOREM 1.1 ([28]). Let $M$ be a locally strongly convex affine hypersphere in $\mathbf{R}^{n+1}$ with constant sectional curvature. Then $M$ is locally affine equivalent to either a hyperquadric or the hyperbolic affine hypersphere

$$
x_{1} x_{2} \cdots x_{n+1}=1,
$$

where $\left(x_{1}, \ldots, x_{n+1}\right)$ is the standard coordinate of $\mathbf{R}^{n+1}$.

THEOREM 1.2 ([29]). Let $M$ be an affine hypersphere in $\mathbf{R}^{n+1}$ with constant sectional curvature $c$ and nonzero Pick invariant. Then $c=0$ and $M$ is locally affine equivalent to

$$
\left(x_{1}^{2} \pm x_{2}^{2}\right)\left(x_{3}^{2} \pm x_{4}^{2}\right) \cdots\left(x_{2 m-1}^{2} \pm x_{2 m}^{2}\right)=1
$$

if $n=2 m-1$, or

$$
\left(x_{1}^{2} \pm x_{2}^{2}\right)\left(x_{3}^{2} \pm x_{4}^{2}\right) \cdots\left(x_{2 m-1}^{2} \pm x_{2 m}^{2}\right) x_{2 m+1}=1
$$

if $n=2 m$, where $\left(x_{1}, \ldots, x_{n+1}\right)$ is the standard coordinate of $\mathbf{R}^{n+1}$.

2010 Mathematics Subject Classification. Primary 53A15; Secondary 53B25, 53B30.

Key words and phrases. Affine hypersphere; Locally homogeneous; Constant sectional curvature; Pick invariant.

This research was supported by grants of NSFC-11326072, NSFC-11401173, and the Innovation Team of Henan University of Science and Technology (No. 2015XTD010).

Received February 10, 2015; revised June 3, 2015. 
However, the classification problem becomes much more difficult if the affine metric is indefinite and the Pick invariant vanishes [2]. Up to now, the following problem of L. Vrancken remains open.

Problem 1 ([1]). Classify all affine hyperspheres with indefinite affine metric of constant sectional curvature and vanishing Pick invariant.

We remark from $[6,30]$ that affine hyperspheres with constant sectional curvature and nonzero Pick invariant are homogeneous under unimodular affine transformations. However, affine hyperspheres with constant sectional curvature and vanishing Pick invariant are not necessarily homogeneous [25]. The solution of Problem 1 for dimension 2 and 3 has been obtained in [27] and [2] respectively, but there appear many implicit examples.

On the other hand, different from Euclidean geometry, the class of higher dimensional homogeneous affine hypersurfaces is very large (cf. [6]), and one is far from a complete classification. For homogeneous affine surfaces one obtained the classification [21,22]. The classification of 3-dimensional case has been completed except for Lorentzian affine hyperspheres (cf. [3, 20, 23, $24,25])$. For general dimension only partial results are known, see $[4,5,15]$ for details. In particular for locally strongly convex homogeneous affine hyperspheres, Sasaki [26] reduced the classification to that of homogeneous convex cones. We notice that affine hypersurfaces with parallel cubic form are locally homogeneous affine hyperspheres [8], and recently, such homogeneous affine hyperspheres have been classified for the locally strongly convex case [14], the Lorentzian case [12], 3 and 4-dimensional case [11, 13], and the other subcases $[10,19]$.

Recently, a whole family of homogeneous affine hypersurfaces constructed by M. Eastwood and V. Ezhov in [9], called the generalized Cayley hypersurfaces, were found in [18] with the following properties: they are improper affine hypersphere with flat indefinite affine metric, zero Pick invariant and the difference tensor $K$ satisfying $\nabla^{(\alpha)} K=0$ and $K^{n-1} \neq 0$. For each constant $\alpha \in \mathbf{R}$, it is defined by the graph immersion of $x_{n+1}=\Phi\left(x_{1}, \ldots, x_{n} ; \alpha\right)$, where

$$
\Phi\left(x_{1}, \ldots, x_{n} ; \alpha\right)=\sum_{d=2}^{n+1} \frac{(-1)^{d}}{d !} \prod_{s=0}^{d-3}[(1-\alpha) s+2] \sum_{j_{1}+\cdots+j_{d}=n+1} x_{j_{1}} \cdots x_{j_{d}},
$$

and $\left(x_{1}, \ldots, x_{n+1}\right)$ is the standard coordinate of $\mathbf{R}^{n+1}$. This is the Cayley surface, the Cayley hypersurface (1) of [9] and the hypersurface (6.3) of [7] corresponding to $n=2, \alpha=0$ and $\alpha=1$, respectively. Note that the 3-dimensional generalized Cayley hypersurfaces, explicitly given by

$$
x_{4}=\Phi\left(x_{1}, x_{2}, x_{3} ; \alpha\right)=x_{1} x_{3}+\frac{1}{2} x_{2}^{2}-x_{1}^{2} x_{2}+\frac{3-\alpha}{12} x_{1}^{4},
$$

are characterized by $\mathrm{M}$. Ooguri as follows: 
THEOREM 1.3 (cf. Proposition 4.2 of [25]). Let $M^{3}$ be a locally homogeneous affine hypersphere in $\mathbf{R}^{4}$ with constant sectional curvature $\kappa$ and vanishing Pick invariant. If $K^{2} \neq 0$, then $\kappa=0$ and $M^{3}$ is locally affine equivalent to the 3-dimensional generalized Cayley hypersurfaces.

Without the condition of homogeneity, both the characterization of the Cayley hypersurface and that of the generalized Cayley hypersurfaces are obtained in [16] and [18], respectively.

Motivated by above facts, it is nature to consider the following problem:

Problem 2. Besides the $n$-dimensional generalized Cayley hypersurfaces, do there exist other examples, which are locally homogeneous affine hypersphere with constant sectional curvature, vanishing Pick invariant and $K^{n-1} \neq 0$ ?

Remark 1.1. For dimension $n=2$, there indeed exists such affine sphere [21], namely $x_{3}=x_{1} x_{2}+\log x_{1}$. However, for $n=3$ it follows from Theorem 1.3 that there doesn't exist such affine hypersphere. namely

In this paper, we give a positive answer to Problem 2 for $n=4$ and 5,

MAIN THEOREM. Let $M^{n}$ be a locally homogeneous affine hypersphere in $\mathbf{R}^{n+1}$ with constant sectional curvature and vanishing Pick invariant. If $K^{n-1} \neq 0$, then $M^{n}$ is an improper affine hypersphere with flat indefinite affine metric for $n \leq 5$. Furthermore, $M^{4}$ is locally affine equivalent to the graph immersions of

$$
x_{5}=\Phi\left(x_{1}, \ldots, x_{4} ; \alpha\right)-\frac{\beta}{4} x_{1}^{4},
$$

and $M^{5}$ is locally affine equivalent to one of the two graph immersions:

$$
\begin{gathered}
x_{6}=\Phi\left(x_{1}, \ldots, x_{5} ; \alpha\right)-\frac{\gamma}{4} x_{1}^{4}-\frac{2 \beta}{3} x_{1}^{3} x_{2}+\frac{(25-11 \alpha) \beta}{75} x_{1}^{5}, \\
x_{6}=\Phi\left(x_{1}, \ldots, x_{5} ; 0\right)-\alpha x_{1}^{3} x_{3}+\frac{\alpha}{2} x_{1}^{2} x_{2}^{2}+\frac{\alpha(\alpha+1)}{2} x_{1}^{4} x_{2}+\frac{\alpha\left(\alpha^{2}-3\right)}{12} x_{1}^{6} \\
-\frac{\gamma}{4} x_{1}^{4}-\frac{2 \beta}{3} x_{1}^{3} x_{2}+\frac{(1-\alpha) \beta}{3} x_{1}^{5},
\end{gathered}
$$

where $\alpha, \beta, \gamma$ are arbitrary constant, and $\Phi\left(x_{1}, \ldots, x_{n} ; \alpha\right)$ is given by (1.1).

This paper is organized as follows. In Section 2, we introduce the theory of local affine hypersurfaces. In Section 3, we study the locally homogeneous affine hyperspheres of Main Theorem to obtain a canonical local frame. The proof of Main Theorem is given in Section 4 for dimension $n=4$ and in Section 5 for dimension $n=5$, respectively. 


\section{Preliminaries}

We briefly recall the theory of local equiaffine hypersurfaces in $[17,22]$. Let $\mathbf{R}^{n+1}$ be the standard $(n+1)$-dimensional real affine space, i.e., $\mathbf{R}^{n+1}$ endowed with the standard flat connection $D$ and its parallel volume form $\omega$, given by the determinant. Let $F: M \hookrightarrow \mathbf{R}^{n+1}$ be a non-degenerate affine hypersurface. It is well known that on such hypersurface there exists a canonical transversal vector field $\xi$ called the affine normal. Then we can write

$$
\begin{aligned}
& D_{X} F_{*}(Y)=F_{*}\left(\nabla_{X} Y\right)+h(X, Y) \xi, \\
& D_{X} \xi=-F_{*}(S X) .
\end{aligned}
$$

This affine normal induces the following invariants on $M$ : the affine connection $\nabla$, the affine metric, or Berwald-Blaschke metric $h$, the affine shape operator $S$ and the cubic form, or Fubini-Pick form $C:=\nabla h$. Moreover, the affine mean curvature of $M$ is defined by $H=\frac{1}{n}$ trace $S$. The hypersurface $M$ is called an affine hypersphere if $S=H i d$, then one easily proves that $H=$ const if $n \geq 2$. $M$ is called a proper affine hypersphere if $H \neq 0$ and an improper affine hypersphere if $H=0$. For a proper affine hypersphere the affine normal satisfies $\xi=-H(F-c)$, where $c$ is a fixed point in $\mathbf{R}^{n+1}$, called the center of $F(M)$, for simplicity, we choose $c$ as the origin of $\mathbf{R}^{n+1}$. For an improper affine hypersphere the affine normal $\xi$ is constant.

The classical Pick-Berwald theorem states that the affine connection coincides with the Levi-Civita connection $\hat{\nabla}$ of affine metric $h$ if and only if the hypersurface is a hyperquadric. For that reason, the difference tensor $K(X, Y):=$ $\nabla_{X} Y-\hat{\nabla}_{X} Y$, related to the cubic form by $C(X, Y, Z)=-2 h(K(X, Y), Z)$, plays a fundamental role in affine differential geometry. Denote by $\hat{R}$ the curvature tensor of $\hat{\nabla}$, by difference tensor $K$ we have the Gauss and Codazzi equations:

$$
\begin{gathered}
\hat{R}(X, Y) Z=\frac{1}{2}[h(Y, Z) S X-h(X, Z) S Y+h(S Y, Z) X-h(S X, Z) Y]-\left[K_{X}, K_{Y}\right] Z \\
\left(\hat{\nabla}_{X} K\right)(Y, Z)-\left(\hat{\nabla}_{Y} K\right)(X, Z)=\frac{1}{2}[h(Y, Z) S X-h(X, Z) S Y \\
-h(S Y, Z) X+h(S X, Z) Y],
\end{gathered}
$$

Contracting Gauss equation twice we have

$$
\chi=H+J
$$

where $J=\frac{1}{n(n-1)} h(K, K)$ is the Pick invariant and $\chi$ is the normalized scalar curvature of $h$. Moreover, we have the apolarity condition $\operatorname{tr} K_{Z}=0$, and the property that $h(K(X, Y), Z)$ is totally symmetric for all $X, Y$ and $Z$.

For an affine hypersphere with constant affine sectional curvature and $J=0$, we have $\chi=H$ and the Gauss and Codazzi equations reduce to 


$$
\begin{aligned}
& \hat{R}(X, Y) Z=H[h(Y, Z) X-h(X, Z) Y], \\
& {\left[K_{X}, K_{Y}\right] Z=0} \\
& \left(\hat{\nabla}_{X} K\right)(Y, Z)=\left(\hat{\nabla}_{Y} K\right)(X, Z) .
\end{aligned}
$$

We prepare the following definitions and lemmas.

Definition 2.1 (cf. Remark 3.3 of [7]). For given positive integer $k$, a $(1, k+1)$-tensor field $K^{k}$ is defined by

$$
K^{k}\left(X_{1}, \ldots, X_{k+1}\right)=K_{X_{1}} \cdots K_{X_{k}} X_{k+1}
$$

for any $X_{1}, \ldots, X_{k+1}$. If $\left[K_{X}, K_{Y}\right]=0$ for all $X$ and $Y$, the tensor field $K^{k}$ is totally symmetric. Hence $K^{k}$ vanishes identically if and only if $\left(K_{v}\right)^{k} v=0$ for all vectors $v$. Denote by $m$ the smallest number such that the symmetric tensor $K^{m}$ is identically zero at the point $p$. Then for any tangent vector $v$ at $p$, we have $\left(K_{v}\right)^{m} v=0$ and there exists a tangent vector $u$ at $p$ such that $h\left(\left(K_{u}\right)^{m-1} u, u\right) \neq 0$.

Lemma 2.1 (cf. Lemma 3.3 of [7]). If $\left[K_{Y}, K_{Z}\right]=0$ for all $Y$ and $Z$, then $K_{X}$ is nilpotent for each $X$. In particular, $K^{n}=0$.

An affine hypersurface $M$ in $\mathbf{R}^{n+1}$ is called locally homogeneous if for all points $p$ and $q$, there exists a neighborhood $U$ of $p$ and an equiaffine transformation $A$ of $\mathbf{R}^{n+1}$, i.e., $A \in S L\left(n+1, \mathbf{R}^{n+1}\right) \ltimes \mathbf{R}^{n+1}$, such that $A(p)=q$ and $A(U) \subset M$. If $U=M$ holds for any $p \in M$, then $M$ is homogeneous. Recall the following

LEMMA 2.2 (cf. Lemma 2.1 of [25]). If $M$ is locally homogeneous affine hypersurface, then for any $p, q \in M$ there exists a neighborhood $U$ of $p$ and $A \in S L\left(n+1, \mathbf{R}^{n+1}\right) \ltimes \mathbf{R}^{n+1}$ such that $A(F(p))=F(q), A(F(U)) \subset F(M)$ and $A_{*}(\xi(p))=\xi(q)$. Such transformation $A$ preserves $\nabla, h, S$ and $K$.

\section{A canonical local frame}

Let $M$ be a locally homogeneous affine hypersphere of $\mathbf{R}^{n+1}$ with constant sectional curvature and zero Pick invariant, i.e., $J=0$. Then we have $\chi=H$, (2.4), (2.5) and (2.6). Moreover, if $K^{n-1} \neq 0$ we can choose a canonical local frame as follows.

Lemma 3.1. If $\left[K_{X}, K_{Y}\right]=0$ for each $X, Y$ and $K^{n-1} \neq 0$, then there exists a local frame $\left\{X_{1}, \ldots, X_{n}\right\}$ such that

$$
K\left(X_{i}, X_{j}\right)=\left\{\begin{array}{ll}
X_{i+j}, & i+j \leq n, \\
0, & \text { otherwise },
\end{array} \quad h\left(X_{i}, X_{j}\right)= \begin{cases}1, & i+j=n+1 \\
0, & \text { otherwise }\end{cases}\right.
$$

The frame is uniquely up to a sign determined. 
Proof. The proof of the first part is the same as the proof of Lemma 6.1 in [7], see also Lemma 3.2 in [16], although we obtain the local frame instead of basis. Note that Lemma 3.1 has been proved for $n=3$ (cf. Lemma 3.4 and Remark 3.4 of [2]). For the uniqueness of frame, we assume that there exist two frames $\left\{X_{1}, \ldots, X_{n}\right\}$ and $\left\{Y_{1}, \ldots, Y_{n}\right\}$ satisfying (3.1). Set $Y_{1}=a_{1} X_{1}+\cdots+$ $a_{n} X_{n}$. Since $Y_{k+1}=K\left(Y_{1}, Y_{k}\right)$ for $k=1, \ldots, n-1$, we see from (3.1) that

$$
\begin{aligned}
h\left(Y_{1}, Y_{\ell}\right) & =h\left(a_{1} X_{1}+\cdots+a_{n} X_{n}, \sum_{i_{1}+\cdots+i_{\ell} \leq n} a_{i_{1}} \cdots a_{i_{\ell}} X_{i_{1}+\cdots+i_{\ell}}\right) \\
& =a_{n+1-\ell} \sum_{i_{1}+\cdots+i_{\ell}=\ell} a_{i_{1}} \cdots a_{i_{\ell}}+\cdots+a_{1} \sum_{i_{1}+\cdots+i_{\ell}=n} a_{i_{1}} \cdots a_{i_{\ell}},
\end{aligned}
$$

where $\ell=2, \ldots, n$. Denote by $\delta$ the standard Kronecker delta. Solving the equations $h\left(Y_{1}, Y_{\ell}\right)=\delta_{n}^{\ell}$ for $\ell=n, \ldots, 1$ respectively, we obtain that

$$
a_{1}^{n+1}=1, \quad a_{2}=a_{3}=\cdots=a_{n}=0 .
$$

Hence $Y_{1}= \pm X_{1}$, then by (3.1) the second conclusion is attained.

Set $\hat{\nabla}_{X_{i}} X_{j}=\sum_{k=1}^{n} \Gamma_{i, j}^{k} X_{k}$ for the frame $\left\{X_{1}, \ldots, X_{n}\right\}$. Then, by local homogeneity of $M$ we see from Lemma 2.2 and 3.1 that $\Gamma_{i, j}^{k}$ are constant. From now on we follow the convention:

$$
i, j, k, \ell \in\{1, \ldots, n\} ; \quad \Gamma_{p, q}^{t}=0 \quad \text { if }\{p, q, t\} \nsubseteq\{1, \ldots, n\} .
$$

From (3.1) we obtain

$$
h\left(\hat{\nabla}_{X_{i}} X_{j}, X_{n-k+1}\right)+h\left(X_{j}, \hat{\nabla}_{X_{i}} X_{n-k+1}\right)=0,
$$

which shows

$$
\Gamma_{i, j}^{k}+\Gamma_{i, n-k+1}^{n-j+1}=0, \quad \forall i, j, k .
$$

In particular, $\Gamma_{i, n-k+1}^{k}=0$. By (2.6) there holds

$$
\left(\hat{\nabla}_{X_{i}} K\right)\left(X_{j}, X_{k}\right)=\left(\hat{\nabla}_{X_{j}} K\right)\left(X_{i}, X_{k}\right)=\left(\hat{\nabla}_{X_{k}} K\right)\left(X_{j}, X_{i}\right) .
$$

A simple computation shows

$$
\begin{aligned}
& \sum_{\ell=1}^{n} \Gamma_{i, j+k}^{\ell} X_{\ell}-\sum_{p=1}^{n-k} \Gamma_{i, j}^{p} X_{p+k}-\sum_{q=1}^{n-j} \Gamma_{i, k}^{q} X_{q+j} \\
& =\sum_{\ell=1}^{n} \Gamma_{j, i+k}^{\ell} X_{\ell}-\sum_{p=1}^{n-k} \Gamma_{j, i}^{p} X_{p+k}-\sum_{q=1}^{n-i} \Gamma_{j, k}^{q} X_{q+i} \\
& =\sum_{\ell=1}^{n} \Gamma_{k, j+i}^{\ell} X_{\ell}-\sum_{p=1}^{n-i} \Gamma_{k, j}^{p} X_{p+i}-\sum_{q=1}^{n-j} \Gamma_{k, i}^{q} X_{q+j} .
\end{aligned}
$$


Then we can rewrite above formulas as

$$
\Gamma_{i, j+k}^{\ell}-\Gamma_{i, j}^{\ell-k}-\Gamma_{i, k}^{\ell-j}=\Gamma_{j, i+k}^{\ell}-\Gamma_{j, i}^{\ell-k}-\Gamma_{j, k}^{\ell-i}=\Gamma_{k, j+i}^{\ell}-\Gamma_{k, j}^{\ell-i}-\Gamma_{k, i}^{\ell-j},
$$

which immediately imply that

$$
\Gamma_{i, j+k}^{\ell}=\Gamma_{j, i+k}^{\ell}=\Gamma_{k, j+i}^{\ell}, \quad \ell \leq \min \{i, j, k\} .
$$

Together with (3.3) there hold

$$
\Gamma_{i, n}^{1}=\Gamma_{j, n-j+i}^{1}=\Gamma_{j, n}^{j-i+1}=0, \quad \forall i, j .
$$

Taking $i=1, k=n$ in (3.4), we have

$$
\Gamma_{1, n}^{\ell-j}=\Gamma_{j, n}^{\ell-1}=\Gamma_{n, j}^{\ell-1}+\Gamma_{n, 1}^{\ell-j}-\Gamma_{n, j+1}^{\ell} .
$$

For $\ell \leq j$ we get $\Gamma_{j, n}^{\ell-1}=\Gamma_{n, j}^{\ell-1}-\Gamma_{n, j+1}^{\ell}=0$. By induction and (3.3) there hold

$$
\Gamma_{j, n}^{\ell}=\Gamma_{n, j}^{\ell}=0, \quad \ell<j .
$$

While for $\ell=j+1 \leq n$, by (3.3) we obtain

$$
0=\Gamma_{j, n}^{j}=\Gamma_{n, j}^{j}+\Gamma_{n, 1}^{1}-\Gamma_{n, j+1}^{j+1} .
$$

Thus $\Gamma_{n, j+1}^{j+1}=(j+1) \Gamma_{n, 1}^{1}$ for $j \leq n-1$. Together with $\Gamma_{n, n}^{n}=-\Gamma_{n, 1}^{1}$ (cf. (3.3)) we see that

$$
\Gamma_{j, n}^{j}=\Gamma_{n, j}^{j}=0, \quad \forall j .
$$
obtain

For $\ell \geq j+2$ we set $\ell=j+m+1$ for $m=1, \ldots, n-j-1$ in (3.7) to

$$
\Gamma_{1, n}^{m+1}=\Gamma_{j, n}^{j+m}=\Gamma_{n, j}^{j+m}+\Gamma_{n, 1}^{m+1}-\Gamma_{n, j+1}^{j+m+1}
$$

which by induction gives that $\Gamma_{n, j+1}^{j+m+1}=(j+1) \Gamma_{n, 1}^{m+1}-j \Gamma_{1, n}^{m+1}$ for $m+j \leq n-1$. Together with $\Gamma_{n, n-m}^{n}=-\Gamma_{n, 1}^{m+1}$ (cf. (3.3)) we get

$$
\Gamma_{1, n}^{m+1}=\frac{n-m+1}{n-m-1} \Gamma_{n, 1}^{m+1}, \quad \Gamma_{n, j+1}^{j+m+1}=\left(1-\frac{2 j}{n-m-1}\right) \Gamma_{n, 1}^{m+1} .
$$

Summing above we have proved the following

LEMMA 3.2.

$$
\begin{aligned}
& \Gamma_{j, n}^{\ell}=\Gamma_{n, j}^{\ell}=0, \quad \ell \leq j, \\
& \Gamma_{n, j+1}^{j+m+1}=\left(1-\frac{2 j}{n-m-1}\right) \Gamma_{n, 1}^{m+1}, \quad \Gamma_{j, n}^{j+m}=\frac{n-m+1}{n-m-1} \Gamma_{n, 1}^{m+1}, \\
& \quad m+j \leq n-1 .
\end{aligned}
$$


Next, taking $i=1, k=n-1$ in (3.4) we have

$$
\begin{aligned}
\delta_{1}^{j} \Gamma_{1, n}^{\ell}-\delta_{n}^{\ell} \Gamma_{1, j}^{1}-\Gamma_{1, n-1}^{\ell-j} & =\Gamma_{j, n}^{\ell}-\delta_{n}^{\ell} \Gamma_{j, 1}^{1}-\Gamma_{j, n-1}^{\ell-1} \\
& =\Gamma_{n-1, j+1}^{\ell}-\Gamma_{n-1, j}^{\ell-1}-\Gamma_{n-1,1}^{\ell-j} .
\end{aligned}
$$

For $\ell \leq j \leq n-1$, by (3.9) we obtain $\Gamma_{j, n-1}^{\ell-1}=\Gamma_{n-1, j}^{\ell-1}-\Gamma_{n-1, j+1}^{\ell}=0$. By induction and (3.3) there hold

$$
\Gamma_{j, n-1}^{\ell}=\Gamma_{n-1, j}^{\ell}=0, \quad \ell<j \leq n-1 .
$$

While for $\ell=j+1 \leq n$ in (3.11), by (3.3) and (3.9) we get

$$
\begin{aligned}
\left(1+\delta_{1}^{j}+\delta_{n-1}^{j}\right) \Gamma_{1, n-1}^{1} & =\Gamma_{j, n-1}^{j}+\delta_{n-1}^{j} \Gamma_{n-1,1}^{1}-\Gamma_{j, n}^{j+1} \\
& =\Gamma_{n-1, j}^{j}+\Gamma_{n-1,1}^{1}-\Gamma_{n-1, j+1}^{j+1} .
\end{aligned}
$$

Then there hold

$$
\begin{array}{ll}
\Gamma_{n-1, j+1}^{j+1}=\Gamma_{n-1, j}^{j}+\Gamma_{n-1,1}^{1}-\left(1+\delta_{1}^{j}+\delta_{n-1}^{j}\right) \Gamma_{1, n-1}^{1}, & j \leq n-1 ; \\
\Gamma_{j, n-1}^{j}=\Gamma_{j, n}^{j+1}+\left(1+\delta_{1}^{j}\right) \Gamma_{1, n-1}^{1}, & j \leq n-2 .
\end{array}
$$

By induction in the first line equations of (3.13), we obtain

$$
\Gamma_{n-1, j+1}^{j+1}=(j+1)\left(\Gamma_{n-1,1}^{1}-\Gamma_{1, n-1}^{1}\right)-\delta_{n-1}^{j} \Gamma_{1, n-1}^{1}, \quad j \leq n-1 .
$$

In particular, if $j=n-1$, by $\Gamma_{n-1, n}^{n}=-\Gamma_{n-1,1}^{1}$ we see that $\Gamma_{n-1,1}^{1}=\Gamma_{1, n-1}^{1}$, thus

$$
\Gamma_{n-1, k}^{k}=0, \quad 2 \leq k \leq n-1 .
$$

Considering the second line equations of (3.13), by (3.3) and (3.10) we have

$$
\Gamma_{j, n-1}^{j}=0, \quad 2 \leq j \leq n-2 .
$$
obtain

When $\ell \geq j+2$, set $\ell=j+m+1$ for $m=1, \ldots, n-j-1$ in (3.11), we

$$
\begin{aligned}
\delta_{1}^{j} \Gamma_{1, n}^{m+2}-\delta_{n-m-1}^{j} \Gamma_{1, n-m-1}^{1}-\Gamma_{1, n-1}^{m+1} & =\Gamma_{j, n}^{j+m+1}-\delta_{n-m-1}^{j} \Gamma_{n-m-1,1}^{1}-\Gamma_{j, n-1}^{j+m} \\
& =\Gamma_{n-1, j+1}^{j+m+1}-\Gamma_{n-1, j}^{j+m}-\Gamma_{n-1,1}^{m+1},
\end{aligned}
$$

which by (3.3) and (3.10) reduce to

$$
\begin{array}{ll}
\Gamma_{n-m-1, n-1}^{n-1}=\Gamma_{1, n-1}^{m+1}-\Gamma_{1, n}^{m+2}-2 \Gamma_{n-m-1,1}^{1}, & m \leq n-3, \\
\Gamma_{j, n-1}^{j+m}=\Gamma_{1, n-1}^{m+1}+\left(1-\delta_{1}^{j}\right) \Gamma_{1, n}^{m+2}, & j<n-m-1, \\
\Gamma_{n-1, j+1}^{j+m+1}=\Gamma_{n-1, j}^{j+m}+\Gamma_{n-1,1}^{m+1}-\Gamma_{1, n-1}^{m+1}+\left(\delta_{1}^{j}+\delta_{n-m-1}^{j}\right) \Gamma_{1, n}^{m+2}, & j \leq n-m-1 .
\end{array}
$$


The last equations imply that

$$
\Gamma_{n-1, j+1}^{j+m+1}=(j+1) \Gamma_{n-1,1}^{m+1}-j \Gamma_{1, n-1}^{m+1}+\left(1+\delta_{n-m-1}^{j}\right) \Gamma_{1, n}^{m+2}, \quad j \leq n-m-1 .
$$

Thus by (3.3) we have $(n-m+1) \Gamma_{n-1,1}^{m+1}+2 \Gamma_{1, n}^{m+2}=(n-m-1) \Gamma_{1, n-1}^{m+1}$ and

$$
\Gamma_{n-1, j+1}^{j+m+1}=\left(1-\frac{2 j}{n-m-1}\right)\left(\Gamma_{n-1,1}^{m+1}+\Gamma_{1, n}^{m+2}\right), \quad j<n-m-1 .
$$

Summing above by Lemma 3.2 we proved the following

LEMMA 3.3 .

$$
\begin{array}{ll}
\Gamma_{n-1,1}^{1}=\Gamma_{1, n-1}^{1}, \quad \Gamma_{n-1, k}^{k}=\Gamma_{k, n-1}^{k}=0, & 2 \leq k \leq n-1 ; \\
\Gamma_{j, n-1}^{\ell}=\Gamma_{n-1, j}^{\ell}=0, & \ell<j ; \\
\Gamma_{j, n-1}^{j+m}=\Gamma_{1, n-1}^{m+1}+\left(1-\delta_{1}^{j}\right) \Gamma_{1, n}^{m+2}, & m+j<n-1 ; \\
\Gamma_{n-m-1, n-1}^{n-1}=\Gamma_{1, n-1}^{m+1}-\Gamma_{1, n}^{m+2}-2 \Gamma_{n-m-1,1}^{1}, & m \leq n-3 ; \\
\Gamma_{n-1, j+1}^{j+m+1}=\left(1-\frac{2 j}{n-m-1}\right)\left(\Gamma_{n-1,1}^{m+1}+\Gamma_{1, n}^{m+2}\right), & m+j<n-1 ; \\
\Gamma_{1, n-1}^{m+1}=\frac{n-m+1}{n-m-1} \Gamma_{n-1,1}^{m+1}+\frac{2}{n-m-1} \Gamma_{1, n}^{m+2}, & m \leq n-2 .
\end{array}
$$

Since $M$ has constant sectional curvature $H$, from (2.4) and the definition of $\hat{R}$ we have

$$
\begin{aligned}
H\left(\delta_{n+1}^{j+k} X_{i}-\delta_{n+1}^{i+k} X_{j}\right) & =\hat{R}\left(X_{i}, X_{j}\right) X_{k} \\
& =\hat{\nabla}_{X_{i}} \hat{\nabla}_{X_{j}} X_{k}-\hat{\nabla}_{X_{j}} \hat{\nabla}_{X_{i}} X_{k}-\hat{\nabla}_{\left[X_{i}, X_{j}\right]} X_{k} \\
& =\sum_{p, q=1}^{n}\left(\Gamma_{j, k}^{p} \Gamma_{i, p}^{q}-\Gamma_{i, k}^{p} \Gamma_{j, p}^{q}+\Gamma_{j, i}^{p} \Gamma_{p, k}^{q}-\Gamma_{i, j}^{p} \Gamma_{p, k}^{q}\right) X_{q} .
\end{aligned}
$$

First, taking $i<j=k=n$ in (3.14), by Lemma 3.2 we have

$$
\delta_{1}^{i} H X_{n}=\sum_{i<p<q \leq n}\left(\Gamma_{i, n}^{p} \Gamma_{n, p}^{q}+\Gamma_{i, n}^{p} \Gamma_{p, n}^{q}-\Gamma_{n, i}^{p} \Gamma_{p, n}^{q}\right) X_{q} .
$$

When $n=2, H=0$. For $n \geq 3$, looking at the components of $X_{n}$ we have

$$
\begin{aligned}
\delta_{1}^{i} H & =\sum_{i<p<n}\left(\Gamma_{i, n}^{p} \Gamma_{n, p}^{n}+\Gamma_{i, n}^{p} \Gamma_{p, n}^{n}-\Gamma_{n, i}^{p} \Gamma_{p, n}^{n}\right) \\
& =\sum_{m=1}^{n-i-1}\left(\Gamma_{i, n}^{i+m} \Gamma_{n, i+m}^{n}+\Gamma_{i, n}^{i+m} \Gamma_{i+m, n}^{n}-\Gamma_{n, i}^{i+m} \Gamma_{i+m, n}^{n}\right) .
\end{aligned}
$$


Note from (3.10) that

$$
\begin{array}{lll}
\Gamma_{n, i}^{i+m}=\left(\frac{n-m+1}{n-m-1}-\frac{2 i}{n-m-1}\right) \Gamma_{n, 1}^{m+1}, & & m+i \leq n ; \\
\Gamma_{i, n}^{i+m}=\frac{n-m+1}{n-m-1} \Gamma_{n, 1}^{m+1}, & & m+i \leq n-1 .
\end{array}
$$

Then (3.16) reduce to

$$
\delta_{1}^{i} H=\sum_{m=1}^{n-i-1} \frac{1}{n-m-1} \Gamma_{n, 1}^{m+1}\left[(n-m+1) \Gamma_{n, i+m}^{n}+2 i \Gamma_{i+m, n}^{n}\right] .
$$

For $i=n-2$, by (3.3) we obtain $\delta_{3}^{n} H=-\frac{1}{n-2} \Gamma_{n, 1}^{2}\left[n \Gamma_{n, 1}^{2}+2(n-2) \Gamma_{n-1,1}^{1}\right]$. The first equation of Lemma 3.3 and (3.17) give $-\Gamma_{n-1,1}^{1}=\Gamma_{1, n}^{2}=\frac{n}{n-2} \Gamma_{n, 1}^{2}$,
thus

$$
\delta_{3}^{n} H=\frac{n}{n-2}\left(\Gamma_{n, 1}^{2}\right)^{2}
$$

Thus $\Gamma_{n, 1}^{2}=\Gamma_{n, n-1}^{n}=\Gamma_{n-1, n}^{n}=0$ for $n \geq 4$, and (3.18) reduce to

$$
\delta_{1}^{i} H=\sum_{m=2}^{n-i-2} \frac{1}{n-m-1} \Gamma_{n, 1}^{m+1}\left[(n-m+1) \Gamma_{n, i+m}^{n}+2 i \Gamma_{i+m, n}^{n}\right] .
$$

For $i=n-3$ in (3.20) we obtain $\delta_{4}^{n} H=0$, i.e., $H=0$ when $n=4$. For $i=n-4$, by (3.3) we have

$$
\delta_{5}^{n} H=-\frac{1}{n-3} \Gamma_{n, 1}^{3}\left[(n-1) \Gamma_{n, 1}^{3}+2(n-4) \Gamma_{n-2,1}^{1}\right], \quad n \geq 5 .
$$

Next, taking $i<j=n-1<k=n$ in (3.14), by Lemma 3.2 and 3.3 we have

$$
\begin{aligned}
\delta_{1}^{i} H X_{n-1}= & \sum_{i<p<q \leq n} \Gamma_{i, n}^{p} \Gamma_{n-1, p}^{q} X_{q}-\Gamma_{n-1, n}^{n} \sum_{i<q \leq n} \Gamma_{i, n}^{q} X_{q} \\
& +\sum_{i<p<q \leq n}\left(\Gamma_{i, n-1}^{p}-\Gamma_{n-1, i}^{p}\right) \Gamma_{p, n}^{q} X_{q} .
\end{aligned}
$$

Looking at the components of $X_{n-1}$ we have

$$
\delta_{1}^{i} H=\sum_{i<p<n-1}\left(\Gamma_{i, n}^{p} \Gamma_{n-1, p}^{n-1}+\Gamma_{i, n-1}^{p} \Gamma_{p, n}^{n-1}-\Gamma_{n-1, i}^{p} \Gamma_{p, n}^{n-1}\right)-\Gamma_{n-1, n}^{n} \Gamma_{i, n}^{n-1} .
$$

For $i=n-2$ we get $\delta_{3}^{n} H=0$. Then (3.19) shows $\Gamma_{n, 1}^{2}=0$ for all $n \geq 3$. Summing above, by previous lemmas we have the following 
LEMMA 3.4 .

$$
\begin{array}{ll}
\Gamma_{n, i}^{i+1}=\Gamma_{i, n}^{i+1}=0, & n \geq 3, \\
\Gamma_{j, n-1}^{\ell}=\Gamma_{n-1, j}^{\ell}=0, & \ell \leq j, \\
H=0, & n \leq 4 .
\end{array}
$$

Now, we continue to consider the totally symmetry of $\hat{\nabla} K$. Taking $(i, k)=$ $(1, n-2)$ in (3.4) we have

$$
\begin{aligned}
\Gamma_{1, n+j-2}^{\ell}-\Gamma_{1, j}^{\ell-n+2}-\Gamma_{1, n-2}^{\ell-j} & =\Gamma_{j, n-1}^{\ell}-\Gamma_{j, 1}^{\ell-n+2}-\Gamma_{j, n-2}^{\ell-1} \\
& =\Gamma_{n-2, j+1}^{\ell}-\Gamma_{n-2, j}^{\ell-1}-\Gamma_{n-2,1}^{\ell-j} .
\end{aligned}
$$

For $\ell \leq j \leq n-2$ in (3.23), by Lemma 3.4 we get

$$
0=\Gamma_{j, n-2}^{\ell-1}=\Gamma_{n-2, j}^{\ell-1}-\Gamma_{n-2, j+1}^{\ell},
$$

which imply that

$$
\Gamma_{j, n-2}^{\ell}=\Gamma_{n-2, j}^{\ell}=0, \quad \ell<j \leq n-1 .
$$

Similarly, for $\ell=j+1 \leq n-1$ in (3.23) there hold

$$
\begin{aligned}
\left(1+\delta_{2}^{j}+\delta_{n-2}^{j}\right) \Gamma_{1, n-2}^{1} & =\Gamma_{j, n-2}^{j}-\Gamma_{j, n-1}^{j+1}+\delta_{n-2}^{j} \Gamma_{n-2,1}^{1} \\
& =\Gamma_{n-2, j}^{j}+\Gamma_{n-2,1}^{1}-\Gamma_{n-2, j+1}^{j+1} .
\end{aligned}
$$

On the one hand, there hold $\Gamma_{n-2,2}^{2}=2 \Gamma_{n-2,1}^{1}-\left(1+\delta_{3}^{n}\right) \Gamma_{1, n-2}^{1}$ and

$$
\Gamma_{n-2, j+1}^{j+1}=(j+1)\left(\Gamma_{n-2,1}^{1}-\Gamma_{1, n-2}^{1}\right)-\delta_{n-2}^{j} \Gamma_{1, n-2}^{1}, \quad 2 \leq j \leq n-2 .
$$

Combining with $\Gamma_{n-2, n-1}^{n-1}=-\Gamma_{n-2,2}^{2}$ we see that

$$
\begin{aligned}
& \Gamma_{n-2,1}^{1}=\Gamma_{1, n-2}^{1}, \quad \Gamma_{n-2,2}^{2}=\left(1-\delta_{3}^{n}\right) \Gamma_{1, n-2}^{1}, \\
& \Gamma_{n-2, j+1}^{j+1}=0, \quad 2 \leq j<n-2 .
\end{aligned}
$$

The first equation of (3.26) and (3.10) give that

$$
\Gamma_{n-2,1}^{1}=\Gamma_{1, n-2}^{1}=-\Gamma_{1, n}^{3}=-\frac{n-1}{n-3} \Gamma_{n, 1}^{3},
$$

then we obtain from (3.21) that $\delta_{5}^{n} H=\frac{(n-1)(n-5)}{(n-3)^{2}}\left(\Gamma_{n, 1}^{3}\right)^{2}$, which show that $H=0$ when $n=5$ and $\Gamma_{n, 1}^{3}=0$ for $n>5$. On the other hand, for $j \leq n-2$ in (3.25) there hold

$$
\Gamma_{j, n-2}^{j}-\Gamma_{j, n-1}^{j+1}=\left(1+\delta_{2}^{j}\right) \Gamma_{1, n-2}^{1}
$$


By the third line equation of Lemma 3.3 and (3.26) we obtain

$$
\Gamma_{j, n-2}^{j}=\delta_{2}^{j} \Gamma_{1, n-2}^{1}, \quad 2 \leq j \leq n-2 .
$$

As before, for $\ell \geq j+2$, set $\ell=j+m+1$ for $m=1, \ldots, n-j-1$ in (3.23), by (3.3) we obtain

$$
\begin{aligned}
\left(\delta_{1}^{j}+\right. & \left.\delta_{n-m-1}^{j}\right) \Gamma_{1, n-1}^{m+2}+\left(\delta_{2}^{j}+\delta_{n-m-2}^{j}\right) \Gamma_{1, n}^{m+3}-\Gamma_{1, n-2}^{m+1} \\
& =\Gamma_{j, n-1}^{j+m+1}-\Gamma_{j, n-2}^{m+j}-\delta_{n-m-2}^{j} \Gamma_{n-m-2,1}^{1}-\delta_{n-m-1}^{j} \Gamma_{n-m-1,1}^{2} \\
& =\Gamma_{n-2, j+1}^{j+m+1}-\Gamma_{n-2, j}^{j+m}-\Gamma_{n-2,1}^{m+1}, \quad m+j \leq n-1 .
\end{aligned}
$$

Summing above, by previous lemmas we have proved the following

Lemma 3.5. There hold (3.27) and

$$
\begin{array}{ll}
\Gamma_{n, i}^{i+2}=\Gamma_{i, n}^{i+2}=0, & n>5, \\
\Gamma_{j, n-2}^{\ell}=\Gamma_{n-2, j}^{\ell}=0, & \ell<j, \\
\Gamma_{n-2,1}^{1}=\Gamma_{1, n-2}^{1}=-\frac{n-1}{n-3} \Gamma_{n, 1}^{3}, & n \geq 4, \\
\Gamma_{n-2, j}^{j}=\Gamma_{j, n-2}^{j}=\delta_{2}^{j} \Gamma_{1, n-2}^{1}, & 2 \leq j \leq n-2, \\
H=0, & n=5 .
\end{array}
$$

\section{The 4-dimensional case}

In this section, for dimension $n=4$ we completely determine the affine hyperspheres by proving the following

THEOREM 4.1. Let $M$ be a locally homogeneous 4-dimensional affine hypersphere of $\mathbf{R}^{5}$ with constant sectional curvature and vanishing Pick invariant. If $K^{3} \neq 0$, then $M$ is an improper affine hypersphere with flat indefinite affine metric, and $M$ is locally affine equivalent to one of the graph immersions of polynomials

$$
x_{5}=\Phi\left(x_{1}, \ldots, x_{4} ; \alpha\right)-\frac{\beta}{4} x_{1}^{4}
$$

where $\alpha, \beta$ are arbitrary constant.

Proof. By Lemma $3.2-3.5$, for $n=4$ we see that $H=0$, thus $\hat{R}=0$. Moreover,

$$
\begin{array}{ll}
\Gamma_{k, p}^{q}+\Gamma_{k, 5-q}^{5-p}=0, & \Gamma_{j, 4}^{i}=\Gamma_{4, j}^{i}=\Gamma_{j, 3}^{i}=\Gamma_{3, j}^{i}=0, \quad i \leq j \leq 4, \\
\Gamma_{i, 4}^{i+1}=\Gamma_{4, i}^{i+1}=0, \quad i \leq 3, & \Gamma_{2,4}^{4}=\Gamma_{1,4}^{3}=\Gamma_{2,3}^{3}=2 \Gamma_{3,3}^{4}=3 \Gamma_{4,1}^{3}, \\
\Gamma_{1,3}^{3}=3 \Gamma_{3,1}^{3}+2 \Gamma_{1,4}^{4}, & 2 \Gamma_{2,3}^{4}=2 \Gamma_{1,3}^{3}+\Gamma_{1,4}^{4}, \quad 3 \Gamma_{2,2}^{4}=2 \Gamma_{1,3}^{4} .
\end{array}
$$


For $(i, j, k)=(1,3,1)$ in $(3.14)$, a direct computation gives

$$
\begin{aligned}
0 & =\hat{R}\left(X_{1}, X_{3}\right) X_{1} \\
& =9\left(\Gamma_{4,1}^{3}\right)^{2} X_{1}+\frac{3}{4} \Gamma_{4,1}^{3}\left(16 \Gamma_{3,1}^{3}+11 \Gamma_{1,4}^{4}\right) X_{2}+\Gamma_{3,1}^{3}\left(\Gamma_{3,1}^{3}+\Gamma_{1,4}^{4}\right) X_{3} .
\end{aligned}
$$

Thus $\Gamma_{4,1}^{3}=0$ and $\Gamma_{3,1}^{3}\left(\Gamma_{3,1}^{3}+\Gamma_{1,4}^{4}\right)=0$. Now, taking $(i, j, k)=(1,2,1)$ in (3.14) we further obtain

$$
0=\hat{R}\left(X_{1}, X_{2}\right) X_{1}=\frac{3}{4}\left(6 \Gamma_{3,1}^{3}+5 \Gamma_{1,4}^{4}\right)\left(2 \Gamma_{3,1}^{3}+\Gamma_{1,4}^{4}\right) X_{2}-\frac{1}{3}\left(8 \Gamma_{3,1}^{3}+5 \Gamma_{1,4}^{4}\right) \Gamma_{1,3}^{4} X_{3} .
$$
These equations show that $\Gamma_{3,1}^{3}=\Gamma_{1,4}^{4}=0$. Set $\Gamma_{1,3}^{4}=\frac{\alpha}{2}$ and $\Gamma_{1,2}^{4}=\beta$, there
hold

$$
\begin{aligned}
& \hat{\nabla}_{X_{4}} X_{i}=\hat{\nabla}_{X_{i}} X_{4}=\hat{\nabla}_{X_{3}} X_{i}=\hat{\nabla}_{X_{2}} X_{3}=0, \quad \forall i, \\
& \hat{\nabla}_{X_{1}} X_{3}=\frac{\alpha}{2} X_{4}, \quad \hat{\nabla}_{X_{1}} X_{2}=\beta X_{4}, \quad \hat{\nabla}_{X_{2}} X_{1}=-\frac{\alpha}{3} X_{3}, \\
& \hat{\nabla}_{X_{1}} X_{1}=-\frac{\alpha}{2} X_{2}-\beta X_{3}, \quad \hat{\nabla}_{X_{2}} X_{2}=\frac{\alpha}{3} X_{4} .
\end{aligned}
$$

The only nonzero components of $\hat{\nabla} K$ are

$$
\begin{aligned}
& \hat{\nabla} K\left(X_{2}, X_{1}, X_{1}\right)=\alpha X_{4}, \\
& \hat{\nabla} K\left(X_{1}, X_{1}, X_{1}\right)=\alpha X_{3}+3 \beta X_{4},
\end{aligned}
$$

and the only nonzero Lie brackets are

$$
\left[X_{1}, X_{2}\right]=\frac{\alpha}{3} X_{3}+\beta X_{4}, \quad\left[X_{1}, X_{3}\right]=\frac{\alpha}{2} X_{4} .
$$

Now, we look at the following system of differential equations of $\left(\rho_{1}, \rho_{2}\right)$.

$$
\left\{\begin{array}{l}
X_{1}\left(\rho_{1}\right)=0, \quad X_{2}\left(\rho_{1}\right)=\frac{\alpha}{3}, \quad X_{3}\left(\rho_{1}\right)=X_{4}\left(\rho_{1}\right)=0, \\
X_{1}\left(\rho_{2}\right)=-\frac{\alpha}{2} \rho_{1}, \quad X_{2}\left(\rho_{2}\right)=\beta, \quad X_{3}\left(\rho_{2}\right)=\frac{\alpha}{2}, \quad X_{4}\left(\rho_{2}\right)=0 .
\end{array}\right.
$$

A direct computation shows that for $k=1,2$

$$
\left(X_{i} X_{j}-X_{j} X_{i}-\left[X_{i}, X_{j}\right]\right) \rho_{k}=0, \quad \forall i, j .
$$

Hence, for instance by introducing coordinates, it is clear that the system of differential equations (4.3) has a unique solution $\left(\rho_{1}, \rho_{2}\right)$ with initial conditions $\rho_{1}(0)=\rho_{2}(0)=0$. Then, by straightforward computation, using (4.3) we verify the following lemma.

LEMMA 4.1. The linear independent vector fields

$$
Y_{1}=X_{1}+\rho_{1} X_{3}+\rho_{2} X_{4}, \quad Y_{2}=X_{2}, \quad Y_{3}=X_{3}, \quad Y_{4}=X_{4}
$$


satisfy $\left[Y_{i}, Y_{j}\right]=0$ for all $i, j . \quad$ Hence, there exist local coordinates $\left\{u_{1}, u_{2}, u_{3}, u_{4}\right\}$ on $M$ such that $\frac{\partial}{\partial u_{i}}=Y_{i}$ for $i=1,2,3,4$ and $\rho_{1}=\frac{\alpha}{3} u_{2}, \rho_{2}=\beta u_{2}+\frac{\alpha}{2} u_{3}$.

Expressing the Levi-Civita connection in terms of the frame $Y_{i}$ we see that

$$
\begin{aligned}
& \hat{\nabla}_{Y_{3}} Y_{3}=\hat{\nabla}_{Y_{2}} Y_{3}=\hat{\nabla}_{Y_{3}} Y_{2}=0, \quad \hat{\nabla}_{Y_{4}} Y_{i}=\hat{\nabla}_{Y_{i}} Y_{4}=0, \quad \forall i, \\
& \hat{\nabla}_{Y_{1}} Y_{1}=-\frac{\alpha}{2} Y_{2}-\beta Y_{3}+\frac{1}{6} \alpha^{2} u_{2} Y_{4}, \quad \hat{\nabla}_{Y_{2}} Y_{2}=\frac{\alpha}{3} Y_{4}, \\
& \hat{\nabla}_{Y_{1}} Y_{2}=\hat{\nabla}_{Y_{2}} Y_{1}=\beta Y_{4}, \quad \hat{\nabla}_{Y_{1}} Y_{3}=\hat{\nabla}_{Y_{3}} Y_{1}=\frac{\alpha}{2} Y_{4} .
\end{aligned}
$$

Also the only nonzero components of the affine metric are

$$
h\left(Y_{1}, Y_{1}\right)=2 \beta u_{2}+\alpha u_{3}, \quad h\left(Y_{1}, Y_{2}\right)=\frac{\alpha}{3} u_{2}, \quad h\left(Y_{1}, Y_{4}\right)=h\left(Y_{2}, Y_{3}\right)=1,
$$

and the only nonzero components of the difference tensor are

$$
K_{Y_{1}} Y_{1}=Y_{2}+\frac{2}{3} \alpha u_{2} Y_{4}, \quad K_{Y_{1}} Y_{2}=Y_{3}, \quad K_{Y_{1}} Y_{3}=K_{Y_{2}} Y_{2}=Y_{4}
$$

Note that the affine normal field $\xi$ of improper affine hypersphere $M$ is constant. From $D_{X} Y=K_{X} Y+\hat{\nabla}_{X} Y+h(X, Y) \xi$, it follows that the immersion $F$ is determined by the following system of differential equations:

$$
\left\{\begin{array}{l}
F_{u_{1} u_{1}}=\frac{2-\alpha}{2} F_{u_{2}}-\beta F_{u_{3}}+\frac{\alpha(4+\alpha)}{6} u_{2} F_{u_{4}}+\left(2 \beta u_{2}+\alpha u_{3}\right) \xi \\
F_{u_{1} u_{2}}=F_{u_{3}}+\beta F_{u_{4}}+\frac{\alpha}{3} u_{2} \xi, \quad F_{u_{1} u_{3}}=\frac{2+\alpha}{2} F_{u_{4}}, \\
F_{u_{2} u_{2}}=\frac{3+\alpha}{3} F_{u_{4}}, \quad F_{u_{1} u_{4}}=F_{u_{2} u_{3}}=\xi \\
F_{u_{3} u_{3}}=F_{u_{2} u_{4}}=F_{u_{3} u_{4}}=F_{u_{4} u_{4}}=0,
\end{array}\right.
$$

where $F_{u_{i}}:=F_{*} \frac{\partial}{\partial u_{i}}$. Solving above system of differential equations, up to an affine transformation, we obtain

$$
\begin{aligned}
F=A & +u_{1} A_{1}+\left(u_{2}+\frac{2-\alpha}{4} u_{1}^{2}\right) A_{2}+\left(u_{3}+u_{1} u_{2}+\frac{2-\alpha}{12} u_{1}^{3}-\frac{\beta}{2} u_{1}^{2}\right) A_{3} \\
+ & {\left[u_{4}+\frac{2+\alpha}{2} u_{1} u_{3}+\frac{3+\alpha}{6} u_{2}^{2}+\frac{2+\alpha}{4} u_{1}^{2} u_{2}+\frac{4-\alpha^{2}}{4 ! \times 4} u_{1}^{4}+\beta u_{1} u_{2}-\frac{1}{6} \alpha \beta u_{1}^{3}\right] A_{4} } \\
+ & {\left[u_{1} u_{4}+u_{2} u_{3}+\frac{3+\alpha}{6} u_{1} u_{2}^{2}+\frac{2+\alpha}{4} u_{1}^{2} u_{3}+\frac{2+\alpha}{12} u_{1}^{3} u_{2}\right.} \\
& \left.+\frac{4-\alpha^{2}}{5 ! \times 4} u_{1}^{5}+\frac{\beta}{2} u_{1}^{2} u_{2}-\frac{\alpha \beta}{4 !} u_{1}^{4}\right] \xi
\end{aligned}
$$


where $A_{i}=F_{u_{i}}(0), A=F(0)$ are constant vectors of $\mathbf{R}^{5}$. Because of nondegenerate, $M$ lies linearly full in $\mathbf{R}^{5}$. Hence $\xi, A_{1}, \ldots, A_{4}$ are linearly independent vectors. By an equiaffine transformation we can write

$$
\begin{aligned}
F=( & u_{1}, u_{2}+\frac{2-\alpha}{4} u_{1}^{2}, u_{3}+u_{1} u_{2}+\frac{2-\alpha}{12} u_{1}^{3}-\frac{\beta}{2} u_{1}^{2}, u_{4}+\frac{2+\alpha}{2} u_{1} u_{3}+\frac{3+\alpha}{6} u_{2}^{2} \\
& +\frac{2+\alpha}{4} u_{1}^{2} u_{2}+\frac{4-\alpha^{2}}{4 ! \times 4} u_{1}^{4}+\beta u_{1} u_{2}-\frac{1}{6} \alpha \beta u_{1}^{3}, u_{1} u_{4}+u_{2} u_{3}+\frac{3+\alpha}{6} u_{1} u_{2}^{2} \\
& \left.+\frac{2+\alpha}{4} u_{1}^{2} u_{3}+\frac{2+\alpha}{12} u_{1}^{3} u_{2}+\frac{4-\alpha^{2}}{5 ! \times 4} u_{1}^{5}+\frac{\beta}{2} u_{1}^{2} u_{2}-\frac{\alpha \beta}{4 !} u_{1}^{4}\right) \hookrightarrow \mathbf{R}^{5} .
\end{aligned}
$$

Set $x_{1}=u_{1}, x_{2}=u_{2}+\frac{2-\alpha}{4} u_{1}^{2}, x_{3}=u_{3}+u_{1} u_{2}+\frac{2-\alpha}{12} u_{1}^{3}-\frac{\beta}{2} u_{1}^{2}$ and

$$
\begin{aligned}
x_{4}= & u_{4}+\frac{2+\alpha}{2} u_{1} u_{3}+\frac{3+\alpha}{6} u_{2}^{2}+\frac{2+\alpha}{4} u_{1}^{2} u_{2}+\frac{4-\alpha^{2}}{4 ! \times 4} u_{1}^{4}+\beta u_{1} u_{2}-\frac{1}{6} \alpha \beta u_{1}^{3}, \\
x_{5}= & u_{1} u_{4}+u_{2} u_{3}+\frac{3+\alpha}{6} u_{1} u_{2}^{2}+\frac{2+\alpha}{4} u_{1}^{2} u_{3}+\frac{2+\alpha}{12} u_{1}^{3} u_{2} \\
& +\frac{4-\alpha^{2}}{5 ! \times 4} u_{1}^{5}+\frac{\beta}{2} u_{1}^{2} u_{2}-\frac{\alpha \beta}{4 !} u_{1}^{4},
\end{aligned}
$$

we see that $M$ lies on the graph immersion of polynomial

$$
x_{5}=x_{1} x_{4}+x_{2} x_{3}-x_{1}^{2} x_{3}-x_{1} x_{2}^{2}+\frac{3-\alpha}{3} x_{1}^{3} x_{2}-\frac{(2-\alpha)(3-\alpha)}{30} x_{1}^{5}-\frac{\beta}{4} x_{1}^{4} .
$$

This is exactly the hypersurface (4.1). Obviously, if $\beta=0$ these are exactly the 4-dimensional generalized Cayley hypersurfaces.

\section{The 5-dimensional case}

In this section, for dimension $n=5$ we completely determine the affine hyperspheres by proving the following

THEOREM 5.1. Let $M$ be a locally homogeneous 5-dimensional affine hypersphere of $\mathbf{R}^{6}$ with constant sectional curvature and vanishing Pick invariant. If $K^{4} \neq 0$, then $M$ is an improper affine hypersphere with flat indefinite affine metric, and $M$ is locally affine equivalent to one of the two graph immersions:

$$
\begin{aligned}
x_{6}= & \Phi\left(x_{1}, \ldots, x_{5} ; \alpha\right)-\frac{\gamma}{4} x_{1}^{4}-\frac{2 \beta}{3} x_{1}^{3} x_{2}+\frac{(25-11 \alpha) \beta}{75} x_{1}^{5}, \\
x_{6}= & \Phi\left(x_{1}, \ldots, x_{5} ; 0\right)-\alpha x_{1}^{3} x_{3}+\frac{\alpha}{2} x_{1}^{2} x_{2}^{2}+\frac{\alpha(\alpha+1)}{2} x_{1}^{4} x_{2}+\frac{\alpha\left(\alpha^{2}-3\right)}{12} x_{1}^{6} \\
& -\frac{\gamma}{4} x_{1}^{4}-\frac{2 \beta}{3} x_{1}^{3} x_{2}+\frac{(1-\alpha) \beta}{3} x_{1}^{5},
\end{aligned}
$$

where $\alpha, \beta, \gamma$ are arbitrary constant. 
Proof. By Lemma 3.2-3.5, $H=0$ (thus $\hat{R}=0$ ) and (3.14) we see that

$$
0=h\left(\hat{R}\left(X_{3}, X_{4}\right) X_{3}, X_{2}\right)=\left(\Gamma_{4,3}^{4}\right)^{2},
$$

thus $\Gamma_{4,3}^{4}=0 . \quad$ Moreover,

$$
\begin{array}{ll}
\Gamma_{k, p}^{q}+\Gamma_{k, 6-q}^{6-p}=0, \quad \Gamma_{j, 5}^{i}=\Gamma_{5, j}^{i}=\Gamma_{j, 4}^{i}=\Gamma_{4, j}^{i}=0, \quad i \leq j, \\
\Gamma_{i, 5}^{i+1}=\Gamma_{5, i}^{i+1}=0, \quad i \leq 4, & \Gamma_{j, 3}^{k}=\Gamma_{3, j}^{k}=0, \quad k<j, \\
\Gamma_{i, 4}^{i+1}=\Gamma_{4, i}^{i+1}=0, \quad i \leq 4, & \Gamma_{j, 5}^{j+2}=\Gamma_{5, j}^{j+2}=0, \quad j \leq 3, \\
3 \Gamma_{5,1}^{4}=\Gamma_{1,5}^{4}=\Gamma_{1,4}^{3}+2 \Gamma_{4,3}^{5}, & 2 \Gamma_{2,5}^{5}=\Gamma_{2,4}^{4}+2 \Gamma_{4,3}^{5}, \\
\Gamma_{2,4}^{4}+\Gamma_{2,5}^{5}=2 \Gamma_{1,5}^{4}+\Gamma_{1,4}^{3}=2 \Gamma_{3,3}^{4}+\Gamma_{3,4}^{5}, & 2 \Gamma_{3,4}^{5}=2 \Gamma_{1,4}^{3}+\Gamma_{3,3}^{4}, \\
\Gamma_{1,4}^{4}+\Gamma_{1,5}^{5}=2 \Gamma_{2,4}^{5}-\Gamma_{2,3}^{4}=2 \Gamma_{3,3}^{5}, & \Gamma_{1,4}^{4}=3 \Gamma_{4,1}^{4}+2 \Gamma_{1,5}^{5}, \\
2 \Gamma_{1,4}^{5}=\Gamma_{1,3}^{4}+3 \Gamma_{3,2}^{5} . &
\end{array}
$$

For $(i, j, k)=(2,5,2)$ and $(3,4,2)$ in $(3.14)$, the direct computations show that

$$
\begin{aligned}
& 0=\hat{R}\left(X_{2}, X_{5}\right) X_{2}=\Gamma_{5,2}^{5}\left(\Gamma_{5,2}^{5}+\Gamma_{2,4}^{4}\right) X_{5}, \\
& 0=\hat{R}\left(X_{3}, X_{4}\right) X_{2}=\left(\Gamma_{3,3}^{4} \Gamma_{4,3}^{5}+\Gamma_{5,2}^{5} \Gamma_{4,3}^{5}-\Gamma_{5,2}^{5} \Gamma_{3,4}^{5}\right) X_{5} .
\end{aligned}
$$

Claim: $\Gamma_{5,1}^{4}=0 . \quad$ Otherwise, assume that $\Gamma_{5,1}^{4}=a \neq 0$, by (3.3) we see from the first equation of (5.4) and (5.3) that

$$
\begin{aligned}
& \Gamma_{2,4}^{4}=a, \quad \Gamma_{3,4}^{5}=-\frac{4}{5} a, \quad \Gamma_{3,3}^{4}=\frac{12}{5} a, \quad \Gamma_{1,4}^{3}=-2 a, \\
& \Gamma_{2,5}^{5}=\Gamma_{1,5}^{4}=3 a, \quad \Gamma_{4,3}^{5}=\frac{5}{2} a .
\end{aligned}
$$

Then the second equation of (5.4) implies that $a=0$, a contradiction. Claim has been proved. Then it follows from (5.3) and (5.4) that

$$
\Gamma_{5,1}^{4}=\Gamma_{2,4}^{4}=\Gamma_{3,4}^{5}=\Gamma_{3,3}^{4}=\Gamma_{1,4}^{3}=\Gamma_{2,5}^{5}=\Gamma_{1,5}^{4}=\Gamma_{4,3}^{5}=0 .
$$

Similarly, by (3.3) and the sixth line equations of (5.3) there hold

$$
\begin{aligned}
& 0=h\left(\hat{R}\left(X_{1}, X_{2}\right) X_{1}, X_{4}\right)=\Gamma_{2,4}^{5}\left(\Gamma_{2,4}^{5}-\Gamma_{1,5}^{5}\right) \\
& 0=\hat{R}\left(X_{1}, X_{4}\right) X_{1}=\Gamma_{4,1}^{4}\left(\Gamma_{4,1}^{4}+\Gamma_{1,5}^{5}\right) X_{4}, \\
& 0=\hat{R}\left(X_{1}, X_{3}\right) X_{1}=\Gamma_{3,3}^{5}\left(\Gamma_{3,3}^{5}-\Gamma_{1,5}^{5}\right) X_{3}-\left(\Gamma_{3,3}^{5}+\Gamma_{4,1}^{4}\right)\left(\Gamma_{3,2}^{5}+\Gamma_{1,3}^{4}\right) X_{4} .
\end{aligned}
$$

Claim: $\Gamma_{3,3}^{5}=0$. Otherwise, assume that $\Gamma_{3,3}^{5}=b \neq 0$, by (5.5) we see from the sixth line equations of (5.3) that $\Gamma_{1,5}^{5}=\Gamma_{1,4}^{4}=-3 \Gamma_{4,1}^{4}=b$. Then the second 
HOMOGENEOUS AFFINE HYPERSPHERES WITH CONSTANT SECTIONAL CURVATURE 51 equation of (5.5) implies that $b=0$, a contradiction. Claim has been proved. Moreover, we see from

$$
\left(\hat{\nabla}_{X_{1}} K\right)\left(X_{2}, X_{2}\right)=\left(\hat{\nabla}_{X_{2}} K\right)\left(X_{1}, X_{2}\right), \quad\left(\hat{\nabla}_{X_{2}} K\right)\left(X_{1}, X_{1}\right)=\left(\hat{\nabla}_{X_{1}} K\right)\left(X_{2}, X_{1}\right)
$$

that

$$
3 \Gamma_{1,4}^{4}=\Gamma_{2,4}^{5}+2 \Gamma_{2,3}^{4}, \quad 2 \Gamma_{2,3}^{5}=\Gamma_{1,4}^{5}+2 \Gamma_{1,3}^{4}, \quad 3 \Gamma_{2,2}^{5}=2 \Gamma_{1,3}^{5} .
$$

It follows from this and the sixth line equations of (5.3) that

$$
\Gamma_{2,3}^{4}=2 \Gamma_{2,4}^{5}:=2 \lambda, \quad \Gamma_{1,4}^{4}=\Gamma_{4,1}^{4}=-\Gamma_{1,5}^{5}=\frac{5}{3} \lambda .
$$

Taking this into the first equation of (5.5) we obtain $\lambda=0$, thus

$$
\Gamma_{2,4}^{5}=\Gamma_{2,3}^{4}=\Gamma_{1,4}^{4}=\Gamma_{4,1}^{4}=\Gamma_{1,5}^{5}=\Gamma_{3,3}^{5}=0 .
$$

Set $\Gamma_{1,3}^{5}=\beta, \Gamma_{1,2}^{5}=\gamma$. Summing above, by the last equation of (5.3) and the last two equations of (5.6) we can express the Levi-Civita connection as follows:

$$
\begin{aligned}
& \hat{\nabla}_{X_{5}} X_{i}=\hat{\nabla}_{X_{i}} X_{5}=\hat{\nabla}_{X_{4}} X_{i}=\hat{\nabla}_{X_{i+1}} X_{4}=\hat{\nabla}_{X_{3}} X_{3}=0, \quad \forall i, \\
& \hat{\nabla}_{X_{1}} X_{4}=\Gamma_{1,4}^{5} X_{5}, \quad \hat{\nabla}_{X_{2}} X_{2}=\frac{2}{3} \beta X_{5}, \\
& \hat{\nabla}_{X_{1}} X_{3}=\Gamma_{1,3}^{4} X_{4}+\beta X_{5}, \quad \hat{\nabla}_{X_{3}} X_{1}=\left(\frac{1}{3} \Gamma_{1,3}^{4}-\frac{2}{3} \Gamma_{1,4}^{5}\right) X_{4}, \\
& \hat{\nabla}_{X_{2}} X_{3}=\left(\Gamma_{1,3}^{4}+\frac{1}{2} \Gamma_{1,4}^{5}\right) X_{5}, \quad \hat{\nabla}_{X_{3}} X_{2}=\left(\frac{2}{3} \Gamma_{1,4}^{5}-\frac{1}{3} \Gamma_{1,3}^{4}\right) X_{5}, \\
& \hat{\nabla}_{X_{1}} X_{2}=-\Gamma_{1,3}^{4} X_{3}+\gamma X_{5}, \quad \hat{\nabla}_{X_{2}} X_{1}=-\left(\Gamma_{1,3}^{4}+\frac{1}{2} \Gamma_{1,4}^{5}\right) X_{3}-\frac{2}{3} \beta X_{4}, \\
& \hat{\nabla}_{X_{1}} X_{1}=-\Gamma_{1,4}^{5} X_{2}-\beta X_{3}-\gamma X_{4} .
\end{aligned}
$$

Combining with the Gauss equation $\hat{R}\left(X_{1}, X_{2}\right) X_{1}=0$ we obtain that

$$
\left(\Gamma_{1,3}^{4}+\Gamma_{1,4}^{5}\right)\left(\Gamma_{1,3}^{4}-\frac{1}{3} \Gamma_{1,4}^{5}\right)=0 .
$$

Hence we can divide our discussion into two cases:

$$
\text { Case I: } \Gamma_{1,4}^{5}=3 \Gamma_{1,3}^{4} \text {, Case II: } \Gamma_{1,4}^{5}=-\Gamma_{1,3}^{4} \text {. }
$$

For Case I, we set $\Gamma_{1,4}^{5}=3 \Gamma_{1,3}^{4}=\frac{3 \alpha}{5}$. We note from (5.7) that the only nonzero components of $\hat{\nabla} K$ are

$$
\begin{array}{ll}
\hat{\nabla} K\left(X_{1}, X_{1}, X_{1}\right)=\alpha X_{3}+2 \beta X_{4}+3 \gamma X_{5}, & \hat{\nabla} K\left(X_{3}, X_{1}, X_{1}\right)=\alpha X_{5}, \\
\hat{\nabla} K\left(X_{2}, X_{1}, X_{1}\right)=\alpha X_{4}+2 \beta X_{5}, & \hat{\nabla} K\left(X_{1}, X_{2}, X_{2}\right)=\alpha X_{5} .
\end{array}
$$


The only nonzero Lie brackets are

$$
\begin{array}{ll}
{\left[X_{1}, X_{4}\right]=\frac{3 \alpha}{5} X_{5}, \quad\left[X_{1}, X_{3}\right]=\frac{8 \alpha}{15} X_{4}+\beta X_{5},} \\
{\left[X_{2}, X_{3}\right]=\frac{\alpha}{6} X_{5}, \quad\left[X_{1}, X_{2}\right]=\frac{3 \alpha}{10} X_{3}+\frac{2}{3} \beta X_{4}+\gamma X_{5} .}
\end{array}
$$

Now, we look at the following system of differential equations of $\left(\rho_{1}, \ldots, \rho_{4}\right)$.

$$
\left\{\begin{array}{l}
X_{1}\left(\rho_{1}\right)=0, \quad X_{2}\left(\rho_{1}\right)=\frac{3 \alpha}{10}, \quad X_{3}\left(\rho_{1}\right)=X_{4}\left(\rho_{1}\right)=X_{5}\left(\rho_{i}\right)=0, \quad \forall i, \\
X_{1}\left(\rho_{2}\right)=-\frac{\alpha}{6} \rho_{1}, \quad X_{2}\left(\rho_{2}\right)=0, \quad X_{3}\left(\rho_{2}\right)=\frac{\alpha}{6}, \quad X_{4}\left(\rho_{2}\right)=0, \\
X_{1}\left(\rho_{3}\right)=-\frac{8 \alpha}{15} \rho_{1}, \quad X_{2}\left(\rho_{3}\right)=\frac{2 \beta}{3}, \quad X_{3}\left(\rho_{3}\right)=\frac{8 \alpha}{15}, \quad X_{4}\left(\rho_{3}\right)=0, \\
X_{1}\left(\rho_{4}\right)=-\left(\beta \rho_{1}+\frac{3 \alpha}{5} \rho_{3}\right), \quad X_{2}\left(\rho_{4}\right)=\gamma-\frac{\alpha}{6} \rho_{1}, \\
X_{3}\left(\rho_{4}\right)=\beta, \quad X_{4}\left(\rho_{4}\right)=\frac{3 \alpha}{5} .
\end{array}\right.
$$

Direct computations show that for $k=1,2,3,4$

$$
\left(X_{i} X_{j}-X_{j} X_{i}-\left[X_{i}, X_{j}\right]\right) \rho_{k}=0, \quad \forall i, j .
$$

Hence, for instance by introducing coordinates, it is clear that the system of differential equations (5.9) has a unique solution $\left(\rho_{1}, \ldots, \rho_{4}\right)$ with initial conditions $\rho_{1}(0)=\cdots=\rho_{4}(0)=0$. Then, by straightforward computation, using (5.7) and (5.9) we verify the following lemma.

\section{LEMMA 5.1. The linear independent vector fields}

$$
\begin{aligned}
& Y_{1}=X_{1}+\rho_{1} X_{3}+\rho_{3} X_{4}+\rho_{4} X_{5}, \quad Y_{2}=X_{2}+\rho_{2} X_{5}, \\
& Y_{3}=X_{3}, \quad Y_{4}=X_{4}, \quad Y_{5}=X_{5}
\end{aligned}
$$

satisfy $\left[Y_{i}, Y_{j}\right]=0$ for all $i, j$. Hence, there exist local coordinates $\left\{u_{1}, \ldots, u_{5}\right\}$ on $M$ such that $\frac{\partial}{\partial u_{i}}=Y_{i}$ and

$$
\rho_{1}=\frac{3 \alpha}{10} u_{2}, \quad \rho_{2}=\frac{\alpha}{6} u_{3}, \quad \rho_{3}=\frac{2 \beta}{3} u_{2}+\frac{8 \alpha}{15} u_{3}, \quad \rho_{4}=-\frac{\alpha^{2}}{40} u_{2}^{2}+\gamma u_{2}+\beta u_{3}+\frac{3 \alpha}{5} u_{4} .
$$

Expressing the Levi-Civita connection in terms of the frame $Y_{i}$ we see that

$$
\begin{aligned}
& \hat{\nabla}_{Y_{3}} Y_{3}=\hat{\nabla}_{Y_{i+1}} Y_{4}=\hat{\nabla}_{Y_{4}} Y_{i+1}=0, \quad \hat{\nabla}_{Y_{5}} Y_{j}=\hat{\nabla}_{Y_{j}} Y_{5}=0, \quad \forall i, j, \\
& \hat{\nabla}_{Y_{1}} Y_{1}=-\frac{3 \alpha}{5} Y_{2}-\beta Y_{3}-\left(\gamma+\frac{\alpha^{2}}{25} u_{2}\right) Y_{4}+\left(\frac{7 \alpha \beta}{10} u_{2}+\frac{21 \alpha^{2}}{50} u_{3}\right) Y_{5},
\end{aligned}
$$




$$
\begin{aligned}
& \hat{\nabla}_{Y_{2}} Y_{1}=\hat{\nabla}_{Y_{1}} Y_{2}=-\frac{\alpha}{5} Y_{3}+\left(\gamma+\frac{\alpha^{2}}{10} u_{2}\right) Y_{5}, \quad \hat{\nabla}_{Y_{2}} Y_{3}=\hat{\nabla}_{Y_{3}} Y_{2}=\frac{\alpha}{2} Y_{5}, \\
& \hat{\nabla}_{Y_{1}} Y_{3}=\hat{\nabla}_{Y_{3}} Y_{1}=\frac{\alpha}{5} Y_{4}+\beta Y_{5}, \quad \hat{\nabla}_{Y_{1}} Y_{4}=\hat{\nabla}_{Y_{4}} Y_{1}=\frac{3 \alpha}{5} Y_{5}, \quad \hat{\nabla}_{Y_{2}} Y_{2}=\frac{2 \beta}{3} Y_{5} .
\end{aligned}
$$

The only nonzero components of the affine metric are

$$
\begin{aligned}
& h\left(Y_{1}, Y_{1}\right)=\frac{\alpha^{2}}{25} u_{2}^{2}+2 \gamma u_{2}+2 \beta u_{3}+\frac{6 \alpha}{5} u_{4}, \quad h\left(Y_{1}, Y_{2}\right)=\frac{2 \beta}{3} u_{2}+\frac{7 \alpha}{10} u_{3}, \\
& h\left(Y_{1}, Y_{3}\right)=\frac{3 \alpha}{10} u_{2}, \quad h\left(Y_{1}, Y_{5}\right)=h\left(Y_{2}, Y_{4}\right)=h\left(Y_{3}, Y_{3}\right)=1,
\end{aligned}
$$

and the only nonzero components of the difference tensor are

$$
\begin{aligned}
& K_{Y_{1}} Y_{1}=Y_{2}+\frac{3 \alpha}{5} u_{2} Y_{4}+\left(\frac{4 \beta}{3} u_{2}+\frac{9 \alpha}{10} u_{3}\right) Y_{5}, \quad K_{Y_{1}} Y_{2}=Y_{3}+\frac{3 \alpha}{10} u_{2} Y_{5}, \\
& K_{Y_{1}} Y_{3}=K_{Y_{2}} Y_{2}=Y_{4}, \quad K_{Y_{1}} Y_{4}=K_{Y_{2}} Y_{3}=Y_{5} .
\end{aligned}
$$

As before, the immersion $F$ is determined by the following system of differential equations:

$$
\left\{\begin{aligned}
F_{u_{1} u_{1}}= & \frac{5-3 \alpha}{5} F_{u_{2}}-\beta F_{u_{3}}+\left[\frac{\alpha(15-\alpha)}{25} u_{2}-\gamma\right] F_{u_{4}} \\
& +\left[\frac{(40+21 \alpha) \beta}{30} u_{2}+\frac{(45+21 \alpha) \alpha}{50} u_{3}\right] F_{u_{5}} \\
& +\left(\frac{\alpha^{2}}{25} u_{2}^{2}+2 \gamma u_{2}+2 \beta u_{3}+\frac{6 \alpha}{5} u_{4}\right) \xi \\
F_{u_{1} u_{2}}= & \frac{5-\alpha}{5} F_{u_{3}}+\left[\frac{\alpha(\alpha+3)}{10} u_{2}+\gamma\right] F_{u_{5}}+\left(\frac{2 \beta}{3} u_{2}+\frac{7 \alpha}{10} u_{3}\right) \xi, \\
F_{u_{1} u_{3}}= & \frac{5+\alpha}{5} F_{u_{4}}+\beta F_{u_{5}}+\frac{3 \alpha}{10} u_{2} \xi, \quad F_{u_{2} u_{2}}=F_{u_{4}}+\frac{2 \beta}{3} F_{u_{5}}, \\
F_{u_{1} u_{4}}= & \frac{5+3 \alpha}{5} F_{u_{5}}, \quad F_{u_{2} u_{3}}=\frac{2+\alpha}{2} F_{u_{5}}, \quad F_{u_{1} u_{5}}=F_{u_{2} u_{4}}=F_{u_{3} u_{3}}=\xi \\
F_{u_{3} u_{4}}= & F_{u_{4} u_{4}}=F_{u_{i+1} u_{5}}=0, \quad \forall i,
\end{aligned}\right.
$$

where $F_{u_{i}}:=F_{*} \frac{\partial}{\partial u_{i}}$. Solving above system of differential equations, up to an affine transformation, we obtain

$$
\begin{aligned}
F= & A+u_{1} A_{1}+\left(u_{2}+\frac{5-3 \alpha}{10} u_{1}^{2}\right) A_{2} \\
& +\left(u_{3}+\frac{5-\alpha}{5} u_{1} u_{2}+\frac{(\alpha-5)(3 \alpha-5)}{3 ! \times 25} u_{1}^{3}-\frac{\beta}{2} u_{1}^{2}\right) A_{3}
\end{aligned}
$$




$$
\begin{aligned}
+ & {\left[u_{4}+\frac{5+\alpha}{5} u_{1} u_{3}+\frac{1}{2} u_{2}^{2}+\frac{25-\alpha^{2}}{50} u_{1}^{2} u_{2}\right.} \\
& \left.+\frac{\left(\alpha^{2}-25\right)(3 \alpha-5)}{5 ! \times 25} u_{1}^{4}-\frac{\gamma}{2} u_{1}^{2}-\frac{(5+\alpha) \beta}{30} u_{1}^{3}\right] A_{4} \\
+ & {\left[u_{5}+\frac{5+3 \alpha}{5} u_{1} u_{4}+\frac{2+\alpha}{2} u_{2} u_{3}+\frac{(3 \alpha+5)(\alpha+5)}{50} u_{1}^{2} u_{3}+\frac{5+3 \alpha}{10} u_{1} u_{2}^{2}\right.} \\
& -\frac{\left(\alpha^{2}-25\right)(3 \alpha+5)}{3 ! \times 125} u_{1}^{3} u_{2}+\frac{\left(\alpha^{2}-25\right)\left(9 \alpha^{2}-25\right)}{5 ! \times 25^{2}} u_{1}^{5}+\beta u_{1} u_{3}+\gamma u_{1} u_{2} \\
& \left.+\frac{\beta}{3} u_{2}^{2}+\frac{(5-\alpha) \beta}{10} u_{1}^{2} u_{2}-\left(\frac{\alpha \gamma}{5}+\frac{\beta^{2}}{3 !}\right) u_{1}^{3}-\frac{\alpha \beta}{15} u_{1}^{4}\right] A_{5} \\
+ & {\left[u_{1} u_{5}+u_{2} u_{4}+\frac{1}{2} u_{3}^{2}+\frac{1}{3 !} u_{2}^{3}+\frac{2+\alpha}{2} u_{1} u_{2} u_{3}+\frac{5+3 \alpha}{10} u_{1}^{2} u_{4}+\frac{3 \alpha+5}{20} u_{1}^{2} u_{2}^{2}\right.} \\
& +\frac{(3 \alpha+5)(\alpha+5)}{3 ! \times 25} u_{1}^{3} u_{3}-\frac{\left(\alpha^{2}-25\right)(3 \alpha+5)}{5 ! \times 25} u_{1}^{4} u_{2}+\frac{\left(\alpha^{2}-25\right)\left(9 \alpha^{2}-25\right)}{6 ! \times 25^{2}} u_{1}^{6} \\
& \left.+\frac{\beta}{2} u_{1}^{2} u_{3}+\frac{\beta}{3} u_{1} u_{2}^{2}+\frac{\gamma}{2} u_{1}^{2} u_{2}+\frac{(5-\alpha) \beta}{30} u_{1}^{3} u_{2}-\left(\frac{\alpha \gamma}{20}+\frac{\beta^{2}}{4 !}\right) u_{1}^{4}-\frac{\alpha \beta}{75} u_{1}^{5}\right] \xi
\end{aligned}
$$

where $A_{i}=F_{u_{i}}(0), A=F(0)$ are constant vectors of $\mathbf{R}^{6}$. Similar to the proof of Theorem 4.1 , by an equiaffine transformation we see that $M$ lies on the graph immersion of polynomial

$$
\begin{aligned}
x_{6}= & x_{1} x_{5}+x_{2} x_{4}+\frac{1}{2} x_{3}^{2}-x_{1}^{2} x_{4}-\frac{1}{3} x_{2}^{3}-2 x_{1} x_{2} x_{3}+\frac{3-\alpha}{3} x_{1}^{3} x_{3}+\frac{3-\alpha}{2} x_{1}^{2} x_{2}^{2} \\
& -\frac{(3-\alpha)(2-\alpha)}{6} x_{1}^{4} x_{2}+\frac{4(3-\alpha)(2-\alpha)(5-3 \alpha)}{6 !} x_{1}^{6} \\
& -\frac{\gamma}{4} x_{1}^{4}-\frac{2 \beta}{3} x_{1}^{3} x_{2}+\frac{(25-11 \alpha) \beta}{75} x_{1}^{5} .
\end{aligned}
$$

This is exactly the hypersurface (5.1). Obviously, if $\beta=\gamma=0$ these are exactly the 5-dimensional generalized Cayley hypersurfaces.

For Case II, we set $\Gamma_{1,4}^{5}=-\Gamma_{1,3}^{4}=\alpha$. We note from (5.7) that the only nonzero components of $\hat{\nabla} K$ are

$$
\begin{array}{ll}
\hat{\nabla} K\left(X_{1}, X_{1}, X_{1}\right)=3 \alpha X_{3}+2 \beta X_{4}+3 \gamma X_{5}, & \hat{\nabla} K\left(X_{3}, X_{1}, X_{1}\right)=3 \alpha X_{5}, \\
\hat{\nabla} K\left(X_{2}, X_{1}, X_{1}\right)=-\alpha X_{4}+2 \beta X_{5}, & \hat{\nabla} K\left(X_{1}, X_{2}, X_{2}\right)=-\alpha X_{5} .
\end{array}
$$

The only nonzero Lie brackets are

$$
\begin{array}{ll}
{\left[X_{1}, X_{4}\right]=\alpha X_{5},} & {\left[X_{1}, X_{3}\right]=\beta X_{5},} \\
{\left[X_{2}, X_{3}\right]=-\frac{3}{2} \alpha X_{5},} & {\left[X_{1}, X_{2}\right]=\frac{\alpha}{2} X_{3}+\frac{2}{3} \beta X_{4}+\gamma X_{5} .}
\end{array}
$$


Now, we look at the following system of differential equations of $\left(\rho_{1}, \ldots, \rho_{4}\right)$.

$$
\left\{\begin{array}{l}
X_{1}\left(\rho_{1}\right)=0, \quad X_{2}\left(\rho_{1}\right)=\frac{\alpha}{2}, \quad X_{3}\left(\rho_{1}\right)=X_{4}\left(\rho_{1}\right)=X_{5}\left(\rho_{i}\right)=0, \quad \forall i \\
X_{1}\left(\rho_{2}\right)=\frac{3 \alpha}{2} \rho_{1}, \quad X_{2}\left(\rho_{2}\right)=0, \quad X_{3}\left(\rho_{2}\right)=-\frac{3 \alpha}{2}, \quad X_{4}\left(\rho_{2}\right)=0 \\
X_{1}\left(\rho_{3}\right)=0, \quad X_{2}\left(\rho_{3}\right)=\frac{2 \beta}{3}, \quad X_{3}\left(\rho_{3}\right)=X_{4}\left(\rho_{3}\right)=0 \\
X_{1}\left(\rho_{4}\right)=-\left(\beta \rho_{1}+\alpha \rho_{3}\right), \quad X_{2}\left(\rho_{4}\right)=\gamma+\frac{3 \alpha}{2} \rho_{1}, \\
X_{3}\left(\rho_{4}\right)=\beta, \quad X_{4}\left(\rho_{4}\right)=\alpha
\end{array}\right.
$$

Direct computations show that for $k=1,2,3,4$

$$
\left(X_{i} X_{j}-X_{j} X_{i}-\left[X_{i}, X_{j}\right]\right) \rho_{k}=0, \quad \forall i, j .
$$

As before, by straightforward computation, using (5.7) and (5.10) we verify the following lemma.

\section{LEMma 5.2. The linear independent vector fields}

$$
\begin{aligned}
& Y_{1}=X_{1}+\rho_{1} X_{3}+\rho_{3} X_{4}+\rho_{4} X_{5}, \quad Y_{2}=X_{2}+\rho_{2} X_{5}, \\
& Y_{3}=X_{3}, \quad Y_{4}=X_{4}, \quad Y_{5}=X_{5}
\end{aligned}
$$

satisfy $\left[Y_{i}, Y_{j}\right]=0$ for all $i, j . \quad$ Hence, there exist local coordinates $\left\{u_{1}, \ldots, u_{5}\right\}$ on $M$ such that $\frac{\partial}{\partial u_{i}}=Y_{i}$ and

$$
\rho_{1}=\frac{\alpha}{2} u_{2}, \quad \rho_{2}=-\frac{3 \alpha}{2} u_{3}, \quad \rho_{3}=\frac{2 \beta}{3} u_{2}, \quad \rho_{4}=\frac{3 \alpha^{2}}{8} u_{2}^{2}+\gamma u_{2}+\beta u_{3}+\alpha u_{4} .
$$

Expressing the Levi-Civita connection in terms of $Y_{i}$ we see that

$$
\begin{aligned}
& \hat{\nabla}_{Y_{3}} Y_{3}=\hat{\nabla}_{Y_{i+1}} Y_{4}=\hat{\nabla}_{Y_{4}} Y_{i+1}=0, \quad \hat{\nabla}_{Y_{5}} Y_{j}=\hat{\nabla}_{Y_{j}} Y_{5}=0, \quad \forall i, j, \\
& \hat{\nabla}_{Y_{1}} Y_{1}=-\alpha Y_{2}-\beta Y_{3}-\left(\gamma+\alpha^{2} u_{2}\right) Y_{4}+\left(\frac{7 \alpha \beta}{6} u_{2}-\frac{3 \alpha^{2}}{2} u_{3}\right) Y_{5}, \\
& \hat{\nabla}_{Y_{2}} Y_{1}=\hat{\nabla}_{Y_{1}} Y_{2}=\alpha Y_{3}+\left(\gamma+\frac{\alpha^{2}}{2} u_{2}\right) Y_{5}, \quad \hat{\nabla}_{Y_{2}} Y_{3}=\hat{\nabla}_{Y_{3}} Y_{2}=-\frac{\alpha}{2} Y_{5}, \\
& \hat{\nabla}_{Y_{1}} Y_{3}=\hat{\nabla}_{Y_{3}} Y_{1}=-\alpha Y_{4}+\beta Y_{5}, \quad \hat{\nabla}_{Y_{1}} Y_{4}=\hat{\nabla}_{Y_{4}} Y_{1}=\alpha Y_{5}, \quad \hat{\nabla}_{Y_{2}} Y_{2}=\frac{2 \beta}{3} Y_{5} .
\end{aligned}
$$

The only nonzero components of the affine metric are 


$$
\begin{aligned}
& h\left(Y_{1}, Y_{1}\right)=\alpha^{2} u_{2}^{2}+2 \gamma u_{2}+2 \beta u_{3}+2 \alpha u_{4}, \quad h\left(Y_{1}, Y_{2}\right)=\frac{2 \beta}{3} u_{2}-\frac{3 \alpha}{2} u_{3}, \\
& h\left(Y_{1}, Y_{3}\right)=\frac{\alpha}{2} u_{2}, \quad h\left(Y_{1}, Y_{5}\right)=h\left(Y_{2}, Y_{4}\right)=h\left(Y_{3}, Y_{3}\right)=1,
\end{aligned}
$$

and the only nonzero components of the difference tensor are

$$
\begin{aligned}
& K_{Y_{1}} Y_{1}=Y_{2}+\alpha u_{2} Y_{4}+\left(\frac{4 \beta}{3} u_{2}+\frac{3 \alpha}{2} u_{3}\right) Y_{5}, \quad K_{Y_{1}} Y_{2}=Y_{3}+\frac{\alpha}{2} u_{2} Y_{5}, \\
& K_{Y_{1}} Y_{3}=K_{Y_{2}} Y_{2}=Y_{4}, \quad K_{Y_{1}} Y_{4}=K_{Y_{2}} Y_{3}=Y_{5} .
\end{aligned}
$$

Then, the immersion $F$ is determined by the system of differential equations:

$$
\left\{\begin{aligned}
F_{u_{1} u_{1}}= & (1-\alpha) F_{u_{2}}-\beta F_{u_{3}}+\left(\alpha u_{2}-\alpha^{2} u_{2}-\gamma\right) F_{u_{4}} \\
& +\left[\frac{(7 \alpha+8) \beta}{6} u_{2}+\frac{3 \alpha(1-\alpha)}{2} u_{3}\right] F_{u_{5}}+\left(\alpha^{2} u_{2}^{2}+2 \gamma u_{2}+2 \beta u_{3}+2 \alpha u_{4}\right) \xi \\
F_{u_{1} u_{2}}= & (1+\alpha) F_{u_{3}}+\left[\frac{\alpha(\alpha+1)}{2} u_{2}+\gamma\right] F_{u_{5}}+\left(\frac{2 \beta}{3} u_{2}-\frac{3 \alpha}{2} u_{3}\right) \xi \\
F_{u_{1} u_{3}}= & (1-\alpha) F_{u_{4}}+\beta F_{u_{5}}+\frac{\alpha}{2} u_{2} \xi, \quad F_{u_{2} u_{2}}=F_{u_{4}}+\frac{2 \beta}{3} F_{u_{5}}, \\
F_{u_{1} u_{4}}= & (1+\alpha) F_{u_{5}}, \quad F_{u_{2} u_{3}}=\frac{2-\alpha}{2} F_{u_{5}}, \quad F_{u_{1} u_{5}}=F_{u_{2} u_{4}}=F_{u_{3} u_{3}}=\xi \\
F_{u_{3} u_{4}}= & F_{u_{4} u_{4}}=F_{u_{i+1} u_{5}}=0, \quad \forall i,
\end{aligned}\right.
$$

where $F_{u_{i}}:=F_{*} \frac{\partial}{\partial u_{i}}$. Solving above system of differential equations, up to an affine transformation, we obtain

$$
\begin{aligned}
F= & +u_{1} A_{1}+\left(u_{2}+\frac{1-\alpha}{2} u_{1}^{2}\right) A_{2}+\left[u_{3}+(1+\alpha) u_{1} u_{2}+\frac{1-\alpha^{2}}{3 !} u_{1}^{3}-\frac{\beta}{2} u_{1}^{2}\right] A_{3} \\
+ & {\left[u_{4}+(1-\alpha) u_{1} u_{3}+\frac{1}{2} u_{2}^{2}+\frac{1-\alpha^{2}}{2} u_{1}^{2} u_{2}\right.} \\
& \left.+\frac{\left(1-\alpha^{2}\right)(1-\alpha)}{4 !} u_{1}^{4}-\frac{\gamma}{2} u_{1}^{2}+\frac{(\alpha-1) \beta}{3 !} u_{1}^{3}\right] A_{4} \\
+ & {\left[u_{5}+(1+\alpha) u_{1} u_{4}+\frac{2-\alpha}{2} u_{2} u_{3}+\frac{1-\alpha^{2}}{2} u_{1}^{2} u_{3}+\frac{1+\alpha}{2} u_{1} u_{2}^{2}\right.} \\
& +\frac{(1+\alpha)\left(1-\alpha^{2}\right)}{3 !} u_{1}^{3} u_{2}+\frac{\left(1-\alpha^{2}\right)^{2}}{5 !} u_{1}^{5}+\beta u_{1} u_{3}+\gamma u_{1} u_{2}+\frac{\beta}{3} u_{2}^{2} \\
& \left.+\frac{(1+\alpha) \beta}{2} u_{1}^{2} u_{2}-\frac{2 \alpha \gamma+\beta^{2}}{3 !} u_{1}^{3}\right] A_{5}
\end{aligned}
$$




$$
\begin{aligned}
& +\left[u_{1} u_{5}+u_{2} u_{4}+\frac{1}{2} u_{3}^{2}+\frac{1}{3 !} u_{2}^{3}+\frac{2-\alpha}{2} u_{1} u_{2} u_{3}+\frac{1+\alpha}{2} u_{1}^{2} u_{4}+\frac{1+\alpha}{4} u_{1}^{2} u_{2}^{2}\right. \\
& +\frac{1-\alpha^{2}}{3 !} u_{1}^{3} u_{3}+\frac{\left(1-\alpha^{2}\right)(1+\alpha)}{4 !} u_{1}^{4} u_{2}+\frac{\left(1-\alpha^{2}\right)^{2}}{6 !} u_{1}^{6}+\frac{\beta}{2} u_{1}^{2} u_{3}+\frac{\beta}{3} u_{1} u_{2}^{2} \\
& \left.\quad+\frac{\gamma}{2} u_{1}^{2} u_{2}+\frac{(1+\alpha) \beta}{3 !} u_{1}^{3} u_{2}-\frac{2 \alpha \gamma+\beta^{2}}{4 !} u_{1}^{4}\right] \xi
\end{aligned}
$$

where $A_{i}=F_{u_{i}}(0), A=F(0)$ are constant vectors of $\mathbf{R}^{6}$. As before, by an equiaffine transformation we see that $M$ lies on the graph immersion of polynomial

$$
\begin{aligned}
x_{6}= & x_{1} x_{5}+x_{2} x_{4}+\frac{1}{2} x_{3}^{2}-x_{1}^{2} x_{4}-\frac{1}{3} x_{2}^{3}-2 x_{1} x_{2} x_{3}+(1-\alpha) x_{1}^{3} x_{3}+\frac{3+\alpha}{2} x_{1}^{2} x_{2}^{2} \\
& -\frac{(1-\alpha)(2+\alpha)}{2} x_{1}^{4} x_{2}+\frac{(1-\alpha)^{2}(2+\alpha)}{12} x_{1}^{6}-\frac{\gamma}{4} x_{1}^{4}-\frac{2 \beta}{3} x_{1}^{3} x_{2}+\frac{(1-\alpha) \beta}{3} x_{1}^{5} .
\end{aligned}
$$

This is exactly the hypersurface (5.2) of Main Theorem. When $\alpha=0$, we note that above graph immersion coincides with that of (5.1).

The proof of Main Theorem follows immediately from that $H=0$ for $n \leq 5$ in Section 3, Theorem 4.1 and 5.1.

Acknowledgement. The author would like to express his sincere thanks to the referee for valuable suggestions to improve the paper.

\section{REFERENCES}

[1] T. Binder and U. Simon, Progress in affine differential geometry-problem list and continued bibliography, Geometry and topology of submanifolds X, World Science Publishing, River Edge, 2000, 1-17.

[2] F. Dillen, M. A. Magid and L. Vrancken, Affine hyperspheres with constant affine sectional curvature, Geometry and topology of submanifolds X, World Science Publishing, River Edge, 2000, 31-53.

[ 3 ] F. Dillen and L. VRancken, The classification of 3-dimensional locally strongly convex homogeneous affine hypersurfaces, Manuscripta Math. 80 (1993), 165-180.

[4] F. Dillen AND L. VRancken, Homogeneous affine hypersurfaces with rank one shape operators, Math. Z. 212 (1993), 61-72.

[5] F. Dillen and L. VRancken, Quasi-umblical, locally strongly convex homogeneous affine hypersurfaces, J. Math. Soc. Japan 46 (1994), 477-502.

[ 6] F. Dillen and L. Vrancken, Calabi-type composition of affine spheres, Diff. Geom. Appl. 4 (1994), 303-328.

[ 7 F. Dillen and L. VRancken, Hypersurfaces with parallel difference tensor, Japan J. Math. 24 (1998), 43-60.

[8] F. Dillen, L. Vrancken and S. Yaprak, Affine hypersurfaces with parallel cubic form, Nagoya Math. J. 135 (1994), 153-164.

[9] M. Eastwood and V. Ezhov, Cayley hypersurfaces, Proc. Steklov Inst. Math. 253 (2006), 221-224. 
[10] R. Hildebrand, Centro-affine hypersurface immersions with parallel cubic form, Beitr. Algebra. Geom. 56 (2015), 593-640.

[11] Z. J. Hu AND C. C. LI, The classification of 3-dimensional Lorentzian affine hypersurfaces with parallel cubic form, Diff. Geom. Appl. 29 (2011), 361-373.

[12] Z. J. Hu, C. C. Li, H. Li AND L. VRANCKEN, Lorentzian affine hypersurfaces with parallel cubic form, Results Math. 59 (2011), 577-620.

[13] Z. J. Hu, C. C. Li, H. Li and L. VRancken, The classification of 4-dimensional nondegenerate affine hypersurfaces with parallel cubic form, J. Geom. Phys. 61 (2011), 2035-2057.

[14] Z. J. Hu, H. Li AND L. VRANCKEN, Locally strongly convex affine hypersurfaces with parallel cubic form, J. Diff. Geom. 87 (2011), 239-307.

[15] Z. J. Hu, C. C. Li AND C. ZhanG, On quasi-umbilical locally strongly convex homogeneous affine hypersurfaces, Diff. Geom. Appl. 33 (2014), 46-74.

[16] Z. J. Hu, C. C. Li AND D. Zhang, A differential geometry characterization of the Cayley hypersurface, Proc. Amer. Math. Soc. 139 (2011), 3697-3706.

[17] A.-M. Li, U. Simon AND G. S. ZHAO, Global affine differential geometry of hypersurfaces, W. de Gruyter, Berlin, 1993.

[18] C. C. Li AND D. Zhang, The generalized Cayley hypersurfaces and their geometrical characterization, Results. Math. 68 (2015), 25-44.

[19] C. C. LI, Affine hypersurfaces with parallel difference tensor relative to affine $\alpha$-connection, J. Geom. Phys. 86 (2014), 81-93.

[20] M. A. MAGID AND L. VRANCKEN, Homogeneous hypersurfaces with nondiagonalisable, rank one shape operators, Soochow J. Math. 21 (1995), 89-105.

[21] K. Nomizu AND T. SASAKi, A new model of unimodular-affinely homogeneous surfaces, Manuscripta Math. 73 (1991), 39-44.

[22] K. Nomizu and T. SASAKI, Affine differential geometry: geometry of affine immersions, Cambridge University Press, 1994.

[23] M. OogurI, The classification of 3-dimensional locally homogeneous Blaschke hypersurfaces with nondiagonalizable affine shape operators, Soochow J. Math. 32 (2006), 379-398.

[24] M. OogurI, The classification of 3-dimensional quasi-umbilical locally homogeneous Blaschke hypersurfaces, Diff. Geom. Appl. 25 (2007), 56-77.

[25] M. OOGURI, Three-dimensional locally homogeneous Lorentzian affine hyperspheres with constant sectional curvature, J. Geom. 104 (2013), 137-152.

[26] T. SASAKI, Hyperbolic affine hyperspheres, Nagoya Math. J. 77 (1980), 107-123.

[27] U. Simon, Local classification of twodimensional affine spheres with constant curvature metric, Diff. Geom. Appl. 1 (1991), 123-132.

[28] L. Vrancken, A.-M. Li AND U. Simon, Affine spheres with constant affine sectional curvature, Math. Z. 206 (1991), 651-658.

[29] L. VRAnCKen, The Magid-Ryan conjecture for equiaffine hyperspheres with constant sectional curvature, J. Diff. Geom. 54 (2000), 99-138.

[30] C. P. WANG, Canonical equiaffine hypersurfaces in $\mathbf{R}^{n+1}$, Math. Z. 214 (1993), 579-592.

Cece $\mathrm{Li}$

SChOOL of Mathematics and Statistics

Henan University of SCIENCE AND TeChNOLOGy

LUOYANG 471023

P. R. CHINA

E-mail: ceceli@haust.edu.cn ceceli@sina.com 